\title{
Compressibility in turbulent MHD and passive scalar transport: mean-field theory
}

\author{
I. ROG A CHEVSKII I, $†$, N. KLEEORI N ${ }^{1,2}$ \\ AND A. B RA N D E N B U R G $\mathbf{~}^{2,3,4}$ \\ ${ }^{1}$ Department of Mechanical Engineering, Ben-Gurion University of the Negev, P. O. Box 653, \\ 84105 Beer-Sheva, Israel \\ ${ }^{2}$ Nordita, KTH Royal Institute of Technology and Stockholm University, Roslagstullsbacken \\ 23, 10691 Stockholm, Sweden \\ ${ }^{3}$ Laboratory for Atmospheric and Space Physics, JILA and Department of Astrophysical and \\ Planetary Sciences, University of Colorado, Boulder, CO 80303, USA \\ ${ }^{4}$ Department of Astronomy, AlbaNova University Center, Stockholm University, SE-10691 \\ Stockholm, Sweden
}

(Received 30 August 2018, Revision: 1.118)

We develop a mean-field theory of compressibility effects in turbulent magnetohydrodynamics and passive scalar transport using the quasi-linear approximation and the spectral $\tau$-approach. We find that compressibility decreases the $\alpha$ effect and the turbulent magnetic diffusivity both at small and large magnetic Reynolds numbers, Rm. Similarly, compressibility decreases the turbulent diffusivity for passive scalars both at small and large Péclet numbers, Pe. On the other hand, compressibility does not affect the effective pumping velocity of the magnetic field for large $\mathrm{Rm}$, but it decreases it for small $\mathrm{Rm}$. Density stratification causes turbulent pumping of passive scalars, but it is found to become weaker with increasing compressibility. No such pumping effect exists for magnetic fields. However, compressibility results in a new passive scalar pumping effect from regions of low to high turbulent intensity both for small and large Péclet numbers. It can be interpreted as compressible turbophoresis of noninertial particles and gaseous admixtures, while the classical turbophoresis effect exists only for inertial particles and causes them to be pumped to regions with lower turbulent intensity.

\section{Introduction}

The generation of magnetic fields by a turbulent flow of a conducting fluid is a fundamental problem which has a number of applications in astrophysics, geophysics, planetary physics, and laboratory experiments (see, e.g., Moffatt 1978; Parker 1979; Krause \& Rädler 1980; Zeldovich et al. 1983; Ruzmaikin et al. 1988; Brandenburg \& Subramanian 2005; Rüdiger et al. 2013). Two types of turbulent dynamos are usually considered: largescale and small-scale dynamos. Magnetic field generation on scales much smaller and much larger than the integral scale of turbulence are described as small-scale dynamo and large-scale dynamo, respectively.

The theory of large-scale dynamos is based on a mean-field approach (Moffatt 1978; Krause \& Rädler 1980) in which the magnetic and velocity fields are decomposed into mean and fluctuating parts. Assuming that the averages obey the Reynolds rules, it follows that the fluctuating parts have zero mean values. Let us furthermore assume that there exists a separation of scales, i.e., the maximum scale of turbulent motions (the turbulent integral scale) is much smaller than the characteristic scales of inhomogeneity

$\dagger$ Email address for correspondence: gary@bgu.ac.il 
of the mean fields. In the framework of the mean-field approach, turbulence effects are described in terms of the mean electromotive force, $\overline{\mathcal{E}}=\overline{\boldsymbol{u} \times \boldsymbol{b}}$ as a function of the large-scale magnetic field $\overline{\boldsymbol{B}}$, where $\boldsymbol{u}$ and $\boldsymbol{b}$ are fluctuations of velocity and magnetic field.

For a number of astrophysical applications, the nonlinear dependence of the function $\overline{\mathcal{E}}(\bar{B})$ has been determined analytically for flows that are incompressible, i.e., div $\boldsymbol{U}=0$ (see, e.g., Rogachevskii \& Kleeorin 2000, 2001, 2004; Rogachevskii et al. 2012; Rüdiger et al. 2013; Rogachevskii et al. 2017), or for low-Mach-number density-stratified flows in the anelastic approximation, $\operatorname{div}(\rho \boldsymbol{U})=0$ (see, e.g., Kleeorin \& Rogachevskii 2003; Rogachevskii \& Kleeorin 2006), where $\rho$ is the fluid density. However, in many astrophysical flows, the Mach number is not small and compressibility effects on the mean electromotive force can be important.

Similar questions associated with compressibility effects arise in turbulent transport of passive scalars, where the mean-field approach allows us to determine the large-scale dynamics of passive scalars in small-scale turbulence (McComb 1990; Zeldovich et al. 1990; Frisch 1995; Zaichik et al. 2008; Monin \& Yaglom 2013). The effects of turbulence on passive scalar transport are described by means of a turbulent flux of passive scalar concentration or particle number density $\boldsymbol{F}=\overline{n \boldsymbol{u}}$, where $n$ is the fluctuation of the particle number density (Csanady 1980; Crowe et al. 2011; Balachandar \& Eaton 2010; Piterbarg \& Ostrovskii 2013). Compressibility effects play a crucial role in particle transport, for example they can cause the formation of spatially inhomogeneous distributions of particles, known as particle clusters. These effects are very important in a range of astrophysical and planetary science applications, where turbulence can be temperature stratified at finite Mach numbers (Armitage 2010; Ruzmaikin et al. 1988; Priest 1982).

For temperature-stratified turbulence, large-scale particle clusters (on scales much larger than the integral scale of the turbulence) are formed due to turbulent thermal diffusion (Elperin et al. 1996, 1997). The effects related to compressibility of the turbulent flow or the particle velocity field, result in a pumping effect of particles in regions of minimum mean fluid temperature. In particular, turbulent thermal diffusion causes a turbulent non-diffusive flux of particles in the direction of the turbulent heat flux, so that particles are accumulated in the vicinity of the mean temperature minimum. The phenomenon of turbulent thermal diffusion has been studied theoretically (Elperin et al. 2000, 2001; Amir et al. 2017), detected in direct numerical simulations (Haugen et al. 2012), different laboratory experiments (Buchholz et al. 2004; Eidelman et al. 2006, 2010; Amir et al. 2017), and atmospheric turbulence with temperature inversions (Sofiev et al. 2009). It was also shown to be important for concentrating dust in protoplanetary discs (Hubbard 2015).

A suppression of turbulent magnetic diffusivity by the compressibility of a random homogeneous flow using the quasi-linear approach (the second-order correlation approximation) was demonstrated by Krause \& Rädler (1980). In particular, Krause \& Rädler (1980) derived an equation for the turbulent magnetic diffusivity, $\eta_{\mathrm{t}}$, for small magnetic Reynolds numbers: $\eta_{\mathrm{t}}=\left(\overline{\psi^{2}}-\overline{\phi^{2}}\right) / 3 \eta$, where $\eta$ is the microscopic magnetic diffusivity and the velocity fluctuations are represented as the sum of vortical and potential parts, $\boldsymbol{u}=\boldsymbol{\nabla} \times \boldsymbol{\psi}+\boldsymbol{\nabla} \phi$. Later, Rädler et al. (2011) determined mean-field diffusivities both for passive scalars and magnetic fields for an irrotational homogeneous deterministic flow, using the quasi-linear approach and the test-field method in direct numerical simulations. They showed that the expression for the turbulent diffusivity of a passive scalar coincides with that of $\eta_{\mathrm{t}}$ for small magnetic Reynolds and Péclet numbers after replacing $\eta$ by the 
molecular diffusion coefficient for the passive scalar. They found that the total mean-field diffusivity in irrotational flows may well be smaller than the molecular diffusivity.

In the present study, a mean-field theory of compressibility effects in turbulent magnetohydrodynamics (MHD) and passive scalar transport is developed for inhomogeneous density-stratified turbulent flows at arbitrary Mach number. We also consider helical turbulence with uniform kinetic helicity. We use the quasi-linear approach, which is valid for small magnetic Reynolds and Péclet numbers, and the spectral tau approach, which is applicable to large magnetic Reynolds and Péclet numbers in fully developed turbulence. This allows us to determine the mean electromotive force and the turbulent flux of particles in compressible density-stratified inhomogeneous turbulence.

\section{Turbulent transport of magnetic field}

In this section we study turbulent transport of a magnetic field in compressible helical inhomogeneous turbulence for small and large magnetic Reynolds numbers.

\subsection{Governing equations}

The magnetic field $\boldsymbol{B}(\boldsymbol{x}, t)$ is governed by the induction equation:

$$
\frac{\partial \boldsymbol{B}}{\partial t}=\boldsymbol{\nabla} \times(\boldsymbol{U} \times \boldsymbol{B}-\eta \boldsymbol{\nabla} \times \boldsymbol{B})
$$

where $\eta$ is the magnetic diffusivity due to the electrical conductivity of the fluid, and fluid velocity $\boldsymbol{U}(\boldsymbol{x}, t)$ is determined by the Navier-Stokes equation,

$$
\rho\left(\frac{\partial}{\partial t}+\boldsymbol{U} \cdot \boldsymbol{\nabla}\right) \boldsymbol{U}-\boldsymbol{\nabla} \cdot\left(2 \nu \rho \mathbf{S}^{(\mathrm{U})}\right)=\frac{1}{\mu_{0}}(\boldsymbol{\nabla} \times \boldsymbol{B}) \times \boldsymbol{B}-\boldsymbol{\nabla} P
$$

where $P(\boldsymbol{x}, t)$ is the fluid pressure, $\mathrm{S}_{i j}^{(\mathrm{U})}=\frac{1}{2}\left(U_{i, j}+U_{j, i}\right)-\frac{1}{3} \delta_{i j} \boldsymbol{\nabla} \cdot \boldsymbol{U}$ are the components of the traceless rate-of-strain-tensor $\mathbf{S}^{(\mathrm{U})}$, commas denote partial differentiation, and $\nu$ is the kinematic viscosity.

We apply a mean-field approach and use Reynolds averaging (Krause \& Rädlen 1980). In the framework of this approach, velocity and magnetic fields, fluid density, and pressure are decomposed into mean (denoted by overbars) and fluctuating parts (lowercase symbols). Ensemble averaging of (2.1) yields an equation for the mean magnetic field $\overline{\boldsymbol{B}}(\boldsymbol{x}, t)$ :

$$
\frac{\partial \overline{\boldsymbol{B}}}{\partial t}=\nabla \times(\overline{\boldsymbol{U}} \times \overline{\boldsymbol{B}}+\overline{\mathcal{E}}-\eta \nabla \times \overline{\boldsymbol{B}}),
$$

where $\overline{\boldsymbol{U}}(\boldsymbol{x}, t)$ is the mean velocity and $\overline{\mathcal{E}}=\overline{\boldsymbol{u} \times \boldsymbol{b}}$ is the mean electromotive force. For simplicity, we consider in this study the case $\overline{\boldsymbol{U}}=\mathbf{0}$. We determine $\overline{\mathcal{E}}$ for compressible inhomogeneous and helical turbulence. The procedure of the derivation of the equation for the mean electromotive force is as follows. The momentum and induction equations for fluctuations are given by

$$
\begin{aligned}
& \bar{\rho} \frac{\partial \boldsymbol{u}}{\partial t}-\boldsymbol{\nabla} \cdot\left(2 \nu \bar{\rho} \mathbf{S}^{(\mathrm{u})}\right)=\frac{1}{\mu_{0}}[(\boldsymbol{b} \cdot \boldsymbol{\nabla}) \overline{\boldsymbol{B}}+(\overline{\boldsymbol{B}} \cdot \boldsymbol{\nabla}) \boldsymbol{b}]-\boldsymbol{\nabla} p_{\mathrm{tot}}+\boldsymbol{u}^{(\mathrm{N})}, \\
& \frac{\partial \boldsymbol{b}}{\partial t}-\eta \Delta \boldsymbol{b}=(\overline{\boldsymbol{B}} \cdot \boldsymbol{\nabla}) \boldsymbol{u}-(\boldsymbol{u} \cdot \boldsymbol{\nabla}) \overline{\boldsymbol{B}}-\overline{\boldsymbol{B}}(\boldsymbol{\nabla} \cdot \boldsymbol{u})+\boldsymbol{b}^{(\mathrm{N})},
\end{aligned}
$$

where $\boldsymbol{u}(\boldsymbol{x}, t)$ and $\boldsymbol{b}(\boldsymbol{x}, t)$ are the fluctuations of velocity and magnetic fields, $\boldsymbol{u}^{(\mathrm{N})}$ and $\boldsymbol{b}^{(\mathrm{N})}$ are terms that are nonlinear in the fluctuations, $p_{\text {tot }}=p+\mu_{0}^{-1}(\overline{\boldsymbol{B}} \cdot \boldsymbol{b})$ are the 
fluctuations of the total pressure, $\mathbf{S}^{(\mathrm{u})} \equiv \mathrm{S}_{i j}^{(\mathrm{u})}=\frac{1}{2}\left(u_{i, j}+u_{j, i}\right)-\frac{1}{3} \delta_{i j} \boldsymbol{\nabla} \cdot \boldsymbol{u}$, and $p(\boldsymbol{x}, t)$ is the fluctuation of the fluid pressure. We rewrite (2.4) and (2.5) in $\boldsymbol{k}$ space. In the following, we derive equations for the mean electromotive force for small and large magnetic Reynolds numbers.

\subsection{Small magnetic Reynolds numbers}

We use a quasi-linear approach (also known in the literature as "first-order smoothing" or "second-order correlation approximation"), which is valid for small hydrodynamic and magnetic Reynolds numbers, Re $=\ell_{0} \sqrt{\overline{\boldsymbol{u}^{2}}} / \nu \ll 1$ and $\mathrm{Rm}=\ell_{0} \sqrt{\overline{\boldsymbol{u}^{2}}} / \eta \ll 1$, respectively. Here $\sqrt{\overline{\overline{\boldsymbol{u}^{2}}}}$ is the characteristic turbulent velocity in the integral turbulent scale $\ell_{0}$. In the high conductivity limit (very small microscopic magnetic diffusivity $\eta$ ), the quasi-linear approach is only valid for small Strouhal numbers, which is defined as the ratio of turbulent correlation time, $\tau_{0}$, to turnover time, $\ell_{0} / \sqrt{\overline{\boldsymbol{u}^{2}}}$. This implies that the quasi-linear approach in this limit is only valid for very short turbulent correlation times. Often (see, e.g., Krause \& Rädler 1980) the quasi-linear approach is applied only to the induction equation and the origin of the velocity field is not discussed. In this case the evolution of the magnetic field is considered for a prescribed velocity field, and formally the validity of the quasi-linear approach only requires small magnetic Reynolds numbers.

\subsubsection{Multi-scale approach}

To determine the mean electromotive force, which is a one-point correlation function, and to take into account small-scale properties of the turbulence, e.g., the turbulent spectrum, one must use two-point correlation functions. For fully developed turbulence, scalings for the turbulent correlation time and energy spectrum are related via the Kolmogorov scalings (McComb 1990; Frisch 1995; Monin \& Yaglom 2013). This is the reason why for calculations of the mean electromotive force in fully developed turbulence, we use instantaneous two-point correlation functions. On the other hand, for a random flow with small Re and Rm, there are no universal scalings for the correlation time and energy spectrum. This is the reason why we have to use non-instantaneous two-point correlation functions in this case.

In the framework of the mean-field approach, we assume that there is a separation of spatial and temporal scales, i.e., $\ell_{0} \ll L_{B}$ and $\tau_{0} \ll t_{B}$, where $L_{B}$ and $t_{B}$ are the characteristic spatial and temporal scales characterizing the variations of the mean magnetic field. The mean fields depend on "slow" variables, while fluctuations depend on "fast" variables. Separation into slow and fast variables is widely used in theoretical physics, and all calculations are reduced to Taylor expansions of all functions using small parameters $\ell_{0} / L_{B}$ and $\tau_{0} / t_{B}$. The findings are further truncated to leading order terms.

Separation to slow and fast variables is performed by means of a standard multiscale approach (Roberts \& Soward 1975). In the framework of this approach, the noninstantaneous two-point second-order correlation function of $\boldsymbol{b}$ and $\boldsymbol{u}$ is written as follows:

$$
\begin{aligned}
\overline{b_{i}\left(\boldsymbol{x}, t_{1}\right) u_{j}\left(\boldsymbol{y}, t_{2}\right)}= & \int d \omega_{1} d \omega_{2} d \boldsymbol{k}_{1} d \boldsymbol{k}_{2} \overline{b_{i}\left(\boldsymbol{k}_{1}, \omega_{1}\right) u_{j}\left(\boldsymbol{k}_{2}, \omega_{2}\right)} \exp \left[i\left(\boldsymbol{k}_{1} \cdot \boldsymbol{x}+\boldsymbol{k}_{2} \cdot \boldsymbol{y}\right)\right. \\
& \left.+i\left(\omega_{1} t_{1}+\omega_{2} t_{2}\right)\right]=\int g_{i j}(\boldsymbol{k}, \omega, t, \boldsymbol{R}) \exp [i \boldsymbol{k} \cdot \boldsymbol{r}+i \omega \tilde{\tau}] d \omega d \boldsymbol{k},(2.6)
\end{aligned}
$$

where

$$
g_{i j}(\boldsymbol{k}, \omega, \boldsymbol{R}, t)=\int \overline{b_{i}\left(\boldsymbol{k}_{1}, \omega_{1}\right) u_{j}\left(\boldsymbol{k}_{2}, \omega_{2}\right)} \exp [i \Omega t+i \boldsymbol{K} \cdot \boldsymbol{R}] d \Omega d \boldsymbol{K}
$$


Here we introduced large-scale variables: $\boldsymbol{R}=(\boldsymbol{x}+\boldsymbol{y}) / 2, \boldsymbol{K}=\boldsymbol{k}_{1}+\boldsymbol{k}_{2}, t=\left(t_{1}+t_{2}\right) / 2$, $\Omega=\omega_{1}+\omega_{2}$, and small-scale variables: $\boldsymbol{r}=\boldsymbol{x}-\boldsymbol{y}, \boldsymbol{k}=\left(\boldsymbol{k}_{1}-\boldsymbol{k}_{2}\right) / 2, \tilde{\tau}=t_{1}-t_{2}$, $\omega=\left(\omega_{1}-\omega_{2}\right) / 2$. This implies that $\omega_{1}=\omega+\Omega / 2, \omega_{2}=-\omega+\Omega / 2, \boldsymbol{k}_{1}=\boldsymbol{k}+\boldsymbol{K} / 2$, and $\boldsymbol{k}_{2}=-\boldsymbol{k}+\boldsymbol{K} / 2$. Mean-fields depend on the large-scale variables, while fluctuations depend on the small-scale variables. We have used here the Fourier transformation:

$$
\Phi(\boldsymbol{x}, t)=\int \Phi(\boldsymbol{k}, \omega) \exp [i(\boldsymbol{k} \cdot \boldsymbol{x}+\omega t)] d \omega d \boldsymbol{k} .
$$

Similarly to (2.6)-(2.7), we obtain

$$
f_{i j}(\boldsymbol{k}, \omega, \boldsymbol{R}, t)=\int \overline{u_{i}\left(\boldsymbol{k}_{1}, \omega_{1}\right) u_{j}\left(\boldsymbol{k}_{2}, \omega_{2}\right)} \exp [i \Omega t+i \boldsymbol{K} \cdot \boldsymbol{R}] d \Omega d \boldsymbol{K} .
$$

After separation into slow and fast variables and calculating the function $g_{i j}(\boldsymbol{k}, \omega, \boldsymbol{R}, t)$, expression (2.6) allows us to determine the cross-helicity tensor in physical space and to calculate the limit of $\boldsymbol{r} \rightarrow \mathbf{0}$ and $\tilde{\tau} \rightarrow 0$, which yields

$$
\overline{b_{i}(\boldsymbol{x}, t) u_{j}(\boldsymbol{x}, t)}=\int g_{i j}(\boldsymbol{k}, \omega, \boldsymbol{R}, t) d \omega d \boldsymbol{k} .
$$

For brevity of notations we omit below the large-scale variables $t$ and $\boldsymbol{R}$ in the correlation functions $f_{i j}(\boldsymbol{k}, \omega, \boldsymbol{R}, t)$ and $g_{i j}(\boldsymbol{k}, \omega, \boldsymbol{R}, t)$.

\subsubsection{Cross-helicity tensor}

In the framework of the quasi-linear approach, we neglect nonlinear terms, but keep molecular dissipative terms in (2.5) for the magnetic fluctuations. This allows us to obtain the solution of (2.5) in the limit of small magnetic Reynolds number. Using this solution, we derive the equation for the correlation function $g_{i j}(\boldsymbol{k}, \omega)$ :

$$
\begin{aligned}
g_{i j}(\boldsymbol{k}, \omega)=G_{\eta}\left\{\bar{B}_{p}\left[i k_{p} f_{i j}+\frac{1}{2} \nabla_{p} f_{i j}-\eta k^{2} G_{\eta} k_{s p} \nabla_{s} f_{i j}\right]-\bar{B}_{i}\left[i k_{p} f_{p j}\right.\right. \\
\left.+\frac{1}{2} \nabla_{p} f_{p j}-\eta k^{2} G_{\eta} k_{s p} \nabla_{s} f_{p j}\right]-\left(\nabla_{s} \bar{B}_{p}\right)\left[\frac{1}{2} k_{p} \frac{\partial f_{i j}}{\partial k_{s}}+\eta k^{2} G_{\eta} k_{s p} f_{i j}\right] \\
\left.+\frac{1}{2}\left(\nabla_{s} \bar{B}_{i}\right)\left[-f_{s j}+k_{p} \frac{\partial f_{p j}}{\partial k_{s}}+2 \eta k^{2} G_{\eta} k_{s p} f_{p j}\right]\right\},
\end{aligned}
$$

where $k_{i j}=k_{i} k_{j} / k^{2}$ and $G_{\eta} \equiv G_{\eta}(\boldsymbol{k}, \omega)=\left(\eta \boldsymbol{k}^{2}+i \omega\right)^{-1}$. The derivation of (2.11) is given in Appendix $\mathrm{A}$. Here, for brevity of notations, we omit the large-scale variables $\boldsymbol{R}$ and $t$ in the mean magnetic field $\bar{B}_{p}$ and correlation functions $g_{i j}$ and $f_{i j}$. When $\overline{\boldsymbol{B}}^{2} / 4 \pi \ll \bar{\rho} \overline{\boldsymbol{u}^{2}}$, the correlation function $f_{i j}(\boldsymbol{k}, \omega)$ in (2.11) should be replaced by the correlation function $f_{i j}^{(0)}(\boldsymbol{k}, \omega)$ given below by (2.12) for the random flow with a zero mean magnetic field, called background flow.

\subsubsection{A model for the background random flow for $R e \ll 1$}

In the next step of the derivation, we need a model for the background random flow with a zero mean magnetic field. We consider a random, statistically stationary, densitystratified, inhomogeneous, compressible and helical background flow, which is determined by the following correlation function in Fourier space:

$$
\begin{aligned}
f_{i j}^{(0)}(\boldsymbol{k}, \omega, \boldsymbol{R})= & \frac{\Phi_{u}(\omega) E(k)}{8 \pi k^{2}\left(1+\sigma_{c}\right)}\left\{\left[\delta_{i j}-k_{i j}+\frac{i}{k^{2}}\left(k_{j} \lambda_{i}-k_{i} \lambda_{j}\right)+2 \sigma_{c} k_{i j}\right.\right. \\
& \left.\left.+\left(1+2 \sigma_{c}\right) \frac{i}{2 k^{2}}\left(k_{i} \nabla_{j}-k_{j} \nabla_{i}\right)\right] \overline{\boldsymbol{u}^{2}}-\frac{i}{k^{2}} \varepsilon_{i j p} k_{p} \chi\right\} .
\end{aligned}
$$


Here $\boldsymbol{\lambda}=-\boldsymbol{\nabla} \ln \bar{\rho}$ characterizes the fluid density stratification, $\delta_{i j}$ is the Kronecker tensor, $\varepsilon_{i j n}$ is the fully antisymmetric Levi-Civita tensor, $\chi=\overline{\boldsymbol{u} \cdot(\boldsymbol{\nabla} \times \boldsymbol{u})}$ is the kinetic helicity, and the parameter

$$
\sigma_{c}=\frac{\overline{(\boldsymbol{\nabla} \cdot \boldsymbol{u})^{2}}}{\overline{(\boldsymbol{\nabla} \times \boldsymbol{u})^{2}}}
$$

is the degree of compressibility of the turbulent velocity field. In (2.12), we neglect higher order effects $\sim \mathrm{O}\left(\lambda^{2} \overline{\boldsymbol{u}^{2}}, \nabla^{2} \overline{\boldsymbol{u}^{2}}, \lambda_{i} \nabla_{i} \overline{\boldsymbol{u}^{2}}\right)$, i.e., we assume that $\ell_{0} \ll H$ and $\ell_{0} \ll L_{B}$, where $\ell_{0}$ is the maximum scale of random motions, $H=|\boldsymbol{\lambda}|^{-1}$ is the mean density variation scale, which is assumed to be constant. This means that in (2.12) we only take into account leading effects, which are linear in stratification $\left(\propto \ell_{0} / H\right)$ and inhomogeneity of turbulence $\left(\propto \ell_{0} / L_{B}\right)$. Generally, stratification also contributes to div $\boldsymbol{u}$, and therefore it contributes to the parameter $\sigma_{c}$. However, this contribution is small $\left[\sim \mathrm{O}\left(\lambda^{2} \overline{\boldsymbol{u}^{2}}\right)\right]$, and neglected in (2.12). This implies that we separate effects of the arbitrary Mach number, characterized by the parameter $\sigma_{c}$, and density stratification, described by $\boldsymbol{\lambda}$. Note that the degree of compressibility, $\sigma_{c}$, depends on the Mach number, but this dependence is not known for arbitrary Mach numbers and has to be determined in direct numerical simulations.

We also do not consider here effects related to the non-uniformity of kinetic helicity $(\propto \nabla \chi)$ or the combined effect of stratification and kinetic helicity $(\propto \lambda \chi)$, because their contributions to the mean electromotive force are much smaller in comparison with the standard contributions to the mean electromotive force caused by the turbulent motions (they are of the order of the terms we neglected in the present study). The non-uniformity of kinetic helicity (Yokoi \& Yoshizawa 1993; Yokoi \& Brandenburg 2016; Kleeorin \& Rogachevskii 2018) or the combined effect of stratification and kinetic helicity (Kleeorin \& Rogachevskii 2018) contribute to the generation of large-scale vorticity or large-scale shear motions.

In (2.12), we assume that helical and non-helical parts of random flow have the same power-law spectrum $E(k)=k_{0}^{-1}(q-1)\left(k / k_{0}\right)^{-q}$ for the wave number range $k_{0}<k<k_{\nu}$, where $k_{\nu}=1 / \ell_{\nu}$ is the wave number based on the viscous scale $\ell_{\nu}$, and $k_{0}=1 / \ell_{0} \ll k_{\nu}$. We assume that there are no random motions for $k<k_{0}$. This implies that $\ell_{0}$ is the maximum scale of random motions, and $E(k)=0$ for $k<k_{0}$. For simplicity we also assume that compressible and incompressible parts of the random flow have the same spectra. We consider the frequency function $\Phi_{u}(\omega)$ in the form of a Lorentz profile: $\Phi_{u}(\omega)=\left[\pi \tau_{0}\left(\omega^{2}+\tau_{0}^{-2}\right)\right]^{-1}$, where $\tau_{0}=\ell_{0} / \sqrt{\overline{\boldsymbol{u}^{2}}}$ is the correlation time and $\sqrt{\overline{\boldsymbol{u}^{2}}}$ is the characteristic turbulent velocity at scale $\ell_{0}$. This model for the frequency function corresponds to the correlation function

$$
\overline{u_{i}(t) u_{j}(t+\tau)} \propto \exp \left(-\tau / \tau_{0}\right)
$$

We take into account that for small magnetic Reynolds numbers, $\tau_{0} \gg\left(\eta k^{2}\right)^{-1}$ for all turbulent scales.

In spite of the large-scale effects caused by stratification $\boldsymbol{\lambda}$ and inhomogeneous turbulence $\nabla \overline{u^{2}}$, the model (2.12) describes a weakly anisotropic approximation. Indeed, the contributions of these effects to $f_{i j}^{(0)}$ are small because $\ell_{0} \ll H$ and $\ell_{0} \ll L_{B}$. Since we consider only linear effects in $\boldsymbol{\lambda}$ and $\boldsymbol{\nabla} \overline{\boldsymbol{u}^{2}}$, the second rank tensor $f_{i j}^{(0)}$ is constructed as a linear combination of symmetric tensors with respect to the indexes $i$ and $j, \delta_{i j}$, $k_{i j}$, non-symmetric tensors: $k_{i} \lambda_{j}, k_{j} \lambda_{i}, k_{i} \nabla_{j} \overline{\boldsymbol{u}^{2}}, k_{j} \nabla_{i} \overline{\boldsymbol{u}^{2}}$, and fully antisymmetric tensor $\varepsilon_{i j p} k_{p}$. To determine unknown coefficients multiplying by these tensors, we use the following conditions in the derivation of (2.12): 
(i) $\int f_{i i}^{(0)}(\boldsymbol{k}, \omega, \boldsymbol{K}) \exp (i \boldsymbol{K} \cdot \boldsymbol{R}) d \boldsymbol{k} d \omega d \boldsymbol{K}=\overline{\boldsymbol{u}^{2}}$;

(ii) $i \varepsilon_{i p j} \int\left(-k_{p}+K_{p} / 2\right) f_{i j}^{(0)}(\boldsymbol{k}, \omega, \boldsymbol{K}) \exp (i \boldsymbol{K} \cdot \boldsymbol{R}) d \boldsymbol{k} d \omega d \boldsymbol{K}=\chi$;

(iii) $f_{i j}^{(0)}(\boldsymbol{k}, \omega, \boldsymbol{K})=f_{j i}^{*(0)}(\boldsymbol{k}, \omega, \boldsymbol{K})=f_{j i}^{(0)}(-\boldsymbol{k}, \omega, \boldsymbol{K})$.

(iv) $\int\left(k_{i}+K_{i} / 2\right)\left(k_{j}-K_{j} / 2\right) f_{i j}^{(0)}(\boldsymbol{k}, \omega, \boldsymbol{K}) \exp (i \boldsymbol{K} \cdot \boldsymbol{R}) d \boldsymbol{k} d \omega d \boldsymbol{K}=\overline{(\operatorname{div} \boldsymbol{u})^{2}}$;

(v) for very low Mach number and when the parameter $\sigma_{c}$ is very small, the continuity equation can be written in the anelastic approximation, $\operatorname{div}(\bar{\rho} \boldsymbol{u})=0$, which implies that $\left(i k_{i}+i K_{i} / 2-\lambda_{i}\right) f_{i j}^{(0)}(\boldsymbol{k}, \omega, \boldsymbol{K})=0$ and $\left(-i k_{j}+i K_{j} / 2-\lambda_{j}\right) f_{i j}^{(0)}(\boldsymbol{k}, \omega, \boldsymbol{K})=0$.

Different contributions to (2.12) have been discussed by Batchelor (1953), Elperin et al. (1995), Rädler et al. (2003), Rogachevskii \& Kleeorin (2018), and Kleeorin \& Rogachevskii (2018). Note that for a purely potential flow, Eq. (2.12) for the background turbulence is used in the limit $\sigma_{c} \rightarrow \infty$, which yields

$$
f_{i j}^{(0)}(\boldsymbol{k}, \omega, \boldsymbol{R})=\frac{\Phi_{u}(\omega) E(k)}{4 \pi k^{2}}\left[k_{i j}+\frac{i}{2 k^{2}}\left(k_{i} \nabla_{j}-k_{j} \nabla_{i}\right)\right] \overline{\boldsymbol{u}^{2}} .
$$

\subsubsection{Mean electromotive force for $\mathrm{Rm} \ll 1$}

The mean electromotive force is given by

$$
\overline{\mathcal{E}}_{i}=\varepsilon_{i n m} \int \overline{b_{m}(\boldsymbol{k}, \omega) u_{n}(-\boldsymbol{k},-\omega)} d \boldsymbol{k} d \omega=\varepsilon_{i n m} \int g_{m n}(\boldsymbol{k}, \omega) d \boldsymbol{k} d \omega .
$$

Integrating in $\omega$ space and $\boldsymbol{k}$ space in (2.16) and neglecting higher order effects $\sim \mathrm{O}\left(\lambda^{2} \overline{\boldsymbol{u}^{2}}\right.$, $\left.\nabla^{2} \overline{\boldsymbol{u}^{2}}, \lambda_{i} \nabla_{i} \overline{\boldsymbol{u}^{2}}\right)$, we arrive at an equation for the mean electromotive force, $\overline{\mathcal{E}}=\overline{\boldsymbol{u} \times \boldsymbol{b}}$, for compressible, density-stratified, inhomogeneous and helical background turbulence:

$$
\overline{\mathcal{E}}=\alpha \overline{\boldsymbol{B}}+\boldsymbol{\gamma} \times \overline{\boldsymbol{B}}-\eta_{\mathrm{t}} \boldsymbol{\nabla} \times \overline{\boldsymbol{B}},
$$

where the $\alpha$ effect, the turbulent magnetic diffusivity $\eta_{\mathrm{t}}$, and the pumping velocity $\gamma$ for $\mathrm{Rm} \ll 1$ are given by

$$
\begin{aligned}
\alpha & =-\frac{(q-1)}{3(q+1)} \frac{\tau_{0} \overline{\boldsymbol{u} \cdot(\boldsymbol{\nabla} \times \boldsymbol{u})}}{1+\sigma_{c}} \mathrm{Rm}, \\
\eta_{\mathrm{t}} & =\frac{(q-1)}{3(q+1)} \tau_{0} \overline{\boldsymbol{u}^{2}}\left(1-\frac{2 \sigma_{c}}{1+\sigma_{c}}\right) \mathrm{Rm} \equiv \frac{(q-1)}{3(q+1)}\left(\frac{1-\sigma_{c}}{1+\sigma_{c}}\right) \mathrm{Rm}^{2} \eta, \\
\gamma & =-\frac{1}{2} \boldsymbol{\nabla} \eta_{\mathrm{t}},
\end{aligned}
$$

and $\tau_{0}=\ell_{0} / \sqrt{\overline{\boldsymbol{u}^{2}}}$. Let us analyze the obtained results. Since $\tau_{0} \mathrm{Rm}=\ell_{0}^{2} / \eta$, the turbulent transport coefficients given by (2.18)-(2.20) are determined only by the resistive time scale, $\ell_{0}^{2} / \eta$. The effective pumping velocity $\gamma$ of the large-scale magnetic field is independent of $\boldsymbol{\lambda}$ (see detailed derivation of (2.20) and discussion in Appendix B). We also have shown that there are no contributions of $\boldsymbol{\lambda}$ to the effective pumping velocity even for strong stratifications (see Appendix B).

Equations (2.18) - (2.20) imply that for small magnetic Reynolds numbers, compressibility effects characterized by the parameter $\sigma_{c}$ decrease the $\alpha$ effect, turbulent magnetic diffusion, and the effective pumping velocity. However, the total magnetic diffusivity, $\eta+\eta_{\mathrm{t}}$, cannot be negative, because for $\mathrm{Rm} \ll 1$ the magnetic diffusivity, $\eta$, is much larger than the turbulent value, i.e., $\eta \gg\left|\eta_{\mathrm{t}}\right|$. The decrease of turbulent magnetic diffusivity by compressible flows is consistent with the results obtained by Krause \& Rädler (1980) and Rädler et al. (2011) for homogeneous random flow at small magnetic Reynolds numbers. Detailed comparison with these results is discussed in section 4. 
As follows from (2.17)-(2.19), the resulting mean electromotive force $\overline{\mathcal{E}}_{i}$ is determined by the isotropic $\alpha$ effect and turbulent diffusivity. The reason for that is as follows. (i) We consider the case of weak mean magnetic field, i.e., the energy of the mean magnetic field is much smaller than the turbulent kinetic energy. For large mean magnetic field, when the energy of the mean magnetic field is of the order of the turbulent kinetic energy, the $\alpha$ tensor and turbulent diffusivity are anisotropic Rogachevskii \& Kleeorin 2000, 2001). (ii) We do not consider the effects of uniform rotation or large-scale shear. In the case of rotating turbulence (Rädler et al. 2003; Kleeorin \& Rogachevskii 2003) or turbulence with large-scale shear (Rogachevskii \& Kleeorin 2003, 2004), the $\alpha$ tensor and turbulent diffusivity are anisotropic. (iii) For anisotropic background turbulence, e.g., for turbulent convection (Kleeorin \& Rogachevskii| 2003), or for magnetically driven turbulence with relativistic particles (Rogachevskii et al. 2012, 2017), the $\alpha$ tensor and turbulent diffusivity are also anisotropic. (iv) The model (2.12) of background random flow is weakly anisotropic, because $\ell_{0} \ll H$ and $\ell_{0} \ll L_{B}$.

\subsection{Large fluid and magnetic Reynolds numbers}

In this section we consider the case of large fluid and magnetic Reynolds numbers, so that turbulence is fully developed, the Strouhal number is of the order of unity, and the turbulent correlation time is scale-dependent, like in Kolmogorov type turbulence (see, e.g., Monin \& Yaglom 2013; McComb 1990; Frisch 1995). In this case, we perform the Fourier transformation only in $\boldsymbol{k}$ space (but not in $\boldsymbol{\omega}$ space), as is usually done in studies of turbulent transport in a fully developed Kolmogorov-type turbulence. We take into account the nonlinear terms in (2.4) and (2.5) for velocity and magnetic fluctuations and apply the $\tau$ approach.

The $\tau$ approach is a universal tool in turbulent transport for strongly nonlinear systems that allow us to obtain closed results and compare them with the results of laboratory experiments, observations, and numerical simulations (see, e.g., Orszag 1970; Pouquet et al. 1976; Kleeorin et al. 1990; Rogachevskii \& Kleeorin 2004; Brandenburg \& Subramanian 2005; Rogachevskii \& Kleeorin 2007; Rogachevskii et al. 2011). The $\tau$ approximation reproduces many well-known phenomena found by other methods in turbulent transport of particles (see, e.g., Elperin et al. 1996, 1997; Pandya \& Mashayek 2002; Blackman \& Field 2003; Reeks 2005; Sofiev et al. 2009) and magnetic fields, in turbulent convection (see, e.g., Elperin et al. 2002, 2006) and stably stratified turbulent flows (see, e.g., Elperin et al. 2002; Zilitinkevich et al. 2009, 2013) for large fluid and magnetic Reynolds and Péclet numbers. This approach is different from the quasi-linear approach applied in the highconductivity limit. The latter approach is only valid for small Strouhal numbers, i.e., for very short correlation time. Therefore, the final results found with this approach are different from those obtained using the $\tau$ approach.

Using (2.5) for the magnetic fluctuations $\boldsymbol{b}$ and the Navier-Stokes equation (2.4) for the velocity fluctuations $\boldsymbol{u}$ in Fourier space, we derive an evolution equation for the cross-helicity tensor,

$$
g_{i j}(\boldsymbol{k}, \boldsymbol{R}, t)=\int \overline{b_{i}(\boldsymbol{k}+\boldsymbol{K} / 2, t) u_{j}(-\boldsymbol{k}+\boldsymbol{K} / 2, t)} \exp [i \boldsymbol{K} \cdot \boldsymbol{R}] d \boldsymbol{K},
$$

which depends on the velocity correlation function:

$$
f_{i j}(\boldsymbol{k}, \boldsymbol{R}, t)=\int \overline{u_{i}(\boldsymbol{k}+\boldsymbol{K} / 2, t) u_{j}(-\boldsymbol{k}+\boldsymbol{K} / 2, t)} \exp [i \boldsymbol{K} \cdot \boldsymbol{R}] d \boldsymbol{K} .
$$


The evolution equation for the cross-helicity tensor reads:

$$
\frac{\partial g_{i j}(\boldsymbol{k})}{\partial t}=i(\boldsymbol{k} \cdot \overline{\boldsymbol{B}}) f_{i j}(\boldsymbol{k})-i k_{m} \bar{B}_{i} f_{m j}(\boldsymbol{k})+I_{i j}(\boldsymbol{k})+g_{i j}^{(\mathrm{N})}(\boldsymbol{k}),
$$

where, for brevity of notation, we omit the large-scale variables $t$ and $\boldsymbol{R}$ in the functions $f_{i j}(\boldsymbol{k}, \boldsymbol{R}, t), g_{i j}(\boldsymbol{k}, \boldsymbol{R}, t), g_{i j}^{(\mathrm{N})}(\boldsymbol{k}, \boldsymbol{R}, t)$ and $\overline{\boldsymbol{B}}(\boldsymbol{R}, t)$. Here the term $g_{i j}^{(\mathrm{N})}$

$$
g_{i j}^{(\mathrm{N})}(\boldsymbol{k})=\int\left[\overline{b_{i}\left(\boldsymbol{k}_{1}\right) \frac{\partial u_{j}\left(\boldsymbol{k}_{2}\right)}{\partial t}}+\overline{b_{i}^{(\mathrm{N})}\left(\boldsymbol{k}_{1}\right) u_{j}\left(\boldsymbol{k}_{2}\right)}\right] \exp [i \boldsymbol{K} \cdot \boldsymbol{R}] d \boldsymbol{K}
$$

is determined by the third-order moments appearing due to the nonlinear terms. Note that since the Navier-Stokes equation is nonlinear, it appears only in the third-order moments $g_{i j}^{(\mathrm{N})}$. Here we took into account that the fluid pressure is nonlinear in the fluctuations. For large magnetic and fluid Reynolds numbers, the dissipative terms caused by the kinematic viscosity $\nu$ and magnetic diffusivity $\eta$ in (2.21) are negligibly small in comparison with the nonlinear terms. The term, $I_{i j}$, which contains the large-scale spatial derivatives of both, the mean magnetic field and the turbulent intensity, is given by

$$
I_{i j}(\boldsymbol{k})=\frac{1}{2}\left[(\overline{\boldsymbol{B}} \cdot \boldsymbol{\nabla}) f_{i j}-k_{n}\left(\frac{\partial f_{i j}}{\partial k_{s}} \nabla_{s} \bar{B}_{n}-\frac{\partial f_{n j}}{\partial k_{s}} \nabla_{s} \bar{B}_{i}\right)-\nabla_{n}\left(\bar{B}_{i} f_{n j}\right)\right] .
$$

This term determines the turbulent magnetic diffusivity and effects of inhomogeneous turbulence. The derivation of (2.21) is given in Appendix C] We consider the case of weak mean magnetic fields, i.e., the energy of the mean magnetic field is much less than the turbulent kinetic energy. This implies that in the present study we do not investigate quenching of the turbulent transport coefficients. Therefore, we do not need evolution equations for the second moments of velocity, $\left\langle u_{i} u_{j}\right\rangle$, and magnetic field, $\left\langle b_{i} b_{j}\right\rangle$ (see, e.g., Rogachevskii \& Kleeorin 2000, 2001, 2004). This implies that we consider linear effects in the mean magnetic field. The nonlinear mean-field theory for the mean electromotive force in a turbulent compressible fluid flow is the subject of a separate ongoing study.

\subsection{1. $\tau$ approach}

Equation 2.21) for the second moment includes first-order spatial differential operators $\hat{\mathcal{M}}$ applied to the third-order moments $F^{(\mathrm{III})}$. The problem arises how to close (2.21), i.e., how to express the third-order term $\hat{\mathcal{M}} F^{(\mathrm{III})}$ through the lower moments $F^{(\mathrm{II})}$ Monin \& Yaglom 2013; McComb 1990). We use the spectral $\tau$ approximation which postulates that the deviations of the third-moment terms, $\hat{\mathcal{M}} F^{(\mathrm{III})}(\boldsymbol{k})$, from the contributions to these terms afforded by the background turbulence, $\hat{\mathcal{M}} F^{(\mathrm{III}, 0)}(\boldsymbol{k})$, can be expressed through similar deviations of the second moments, $F^{(\mathrm{II})}(\boldsymbol{k})-F^{(\mathrm{II}, 0)}(\boldsymbol{k})$ :

$$
\hat{\mathcal{M}} F^{(\mathrm{III})}(\boldsymbol{k})-\hat{\mathcal{M}} F^{(\mathrm{III}, 0)}(\boldsymbol{k})=-\frac{1}{\tau_{r}(k)}\left[F^{(\mathrm{II})}(\boldsymbol{k})-F^{(\mathrm{II}, 0)}(\boldsymbol{k})\right],
$$

where $\tau_{r}(k)$ is the scale-dependent relaxation time, which can be identified with the correlation time $\tau(k)$ of the turbulent velocity field for large fluid and magnetic Reynolds numbers (Orszag 1970; Pouquet et al. 1976; Kleeorin et al. 1990; Rogachevskii \& Kleeorin 2004). The functions with the superscript (0) correspond to the background turbulence with zero mean magnetic field. We take into account that $g_{i j}^{(0)}=0$, because when the mean magnetic field is zero, the electromotive force vanishes. We do not take into account magnetic fluctuations caused by a small-scale dynamo (i.e., a dynamo with zero mean magnetic field). Therefore, (2.24) for $g_{i j}(\boldsymbol{k})$ reduces to $\hat{\mathcal{M}} F_{i}^{(\mathrm{III})}(\boldsymbol{k})=-F_{i}(\boldsymbol{k}) / \tau(k)$. Validation of the $\tau$ approximation for different situations has been performed in various numerical simulations and analytical studies Brandenburg \& Subramanian 2005; 
Rogachevskii \& Kleeorin 2007; Rogachevskii et al. 2011, 2012; Brandenburg et al. 2012a; Käpylä et al. 2012).

\subsubsection{Model of background turbulence for $\operatorname{Re} \gg 1$}

In the next step of the derivation we need a model for the background turbulence. We use statistically stationary, density-stratified, inhomogeneous, compressible and helical background turbulence, which is determined by the following correlation function in $\boldsymbol{k}$ space:

$$
\begin{aligned}
f_{i j}^{(0)}(\boldsymbol{k})= & \frac{E(k)}{8 \pi k^{2}\left(1+\sigma_{c}\right)}\left\{\left[\delta_{i j}-k_{i j}+\frac{i}{k^{2}}\left(k_{j} \lambda_{i}-k_{i} \lambda_{j}\right)+2 \sigma_{c} k_{i j}\right.\right. \\
& \left.\left.+\left(1+2 \sigma_{c}\right) \frac{i}{2 k^{2}}\left(k_{i} \nabla_{j}-k_{j} \nabla_{i}\right)\right] \overline{\boldsymbol{u}^{2}}-\frac{i}{k^{2}} \varepsilon_{i j p} k_{p} \chi\right\} .
\end{aligned}
$$

Note that most statements and conditions used for the derivation of the tensorial structure of (2.12) are also valid for (2.25). We assume here that the background turbulence is of Kolmogorov type with constant fluxes of energy and kinetic helicity over the spectrum, i.e., the kinetic energy spectrum for the range of wave numbers $k_{0}<k<k_{\nu}$ is $E(k)=-d \bar{\tau}(k) / d k$, the function $\bar{\tau}(k)=\left(k / k_{0}\right)^{1-q}$ with $1<q<3$ being the exponent of the kinetic energy spectrum ( $q=5 / 3$ for a Kolmogorov spectrum). The condition $q>1$ corresponds to finite kinetic energy for very large fluid Reynolds numbers, while $q<3$ corresponds to finite dissipation of the turbulent kinetic energy at the viscous scale (see, e.g., Monin \& Yaglom 2013; McComb 1990; Frisch 1995). The turbulent correlation time in $\boldsymbol{k}$ space is $\tau(k)=2 \tau_{0} \bar{\tau}(k)$, where $\tau_{0}=\ell_{0} / \sqrt{\overline{\boldsymbol{u}^{2}}}$ is the turbulent correlation time in physical space, and $\sqrt{\overline{\boldsymbol{u}^{2}}}$ is the characteristic turbulent velocity at scale $\ell_{0}$. Note that for fully developed Kolmogorov like turbulence, $\sigma_{c}<1$ (Chassaing et al. 2013).

\subsubsection{Mean electromotive force for $\mathrm{Rm} \gg 1$}

We use the spectral $\tau$ approach and assume that the characteristic time of variation of the mean magnetic field $\bar{B}$ is substantially larger than the correlation time $\tau(k)$ for all turbulence scales. This allows us to get a stationary solution of (2.21). We consider linear effects in the mean magnetic field, so that the function $f_{i j}(\boldsymbol{k})$ in (2.21) and (2.23) should be replaced by $f_{i j}^{(0)}(\boldsymbol{k})$. The mean electromotive force is determined by $\overline{\mathcal{E}}_{i}=$ $\varepsilon_{\text {inm }} \int g_{m n}(\boldsymbol{k}) \mathrm{d} \boldsymbol{k}$. We take into account that the terms with symmetric tensors with respect to the indexes $m$ and $n$ in $g_{m n}(\boldsymbol{k})$ do not contribute to the mean electromotive force. Therefore, the equation for the mean electromotive force is given by

$$
\begin{gathered}
\overline{\mathcal{E}}_{m}=\varepsilon_{m j i} \\
\int \tau(k)\left\{i(\boldsymbol{k} \cdot \overline{\boldsymbol{B}}) f_{i j}^{(0)}-\bar{B}_{i}\left(i k_{n} f_{n j}^{(0)}+\frac{1}{2} \nabla_{n} f_{n j}^{(0)}\right)\right. \\
\left.-\left[f_{n j}^{(0)}+\frac{k}{2}\left(\frac{\mathrm{d} \ln \tau}{\mathrm{d} k}\right) k_{n p} f_{p j}^{(0)}\right] \nabla_{n} \bar{B}_{i}\right\} \mathrm{d} \boldsymbol{k} .
\end{gathered}
$$

Using the model of background turbulence given by (2.25) and integrating in $\boldsymbol{k}$-space (2.26), we arrive at equation for the mean electromotive force (2.17), where the $\alpha$ effect, the turbulent magnetic diffusivity $\eta_{\mathrm{t}}$ and the pumping velocity $\gamma$ for large magnetic Reynolds numbers $(\mathrm{Rm} \gg 1)$ are given by

$$
\begin{aligned}
\alpha & =-\frac{1}{3} \frac{\tau_{0} \overline{\boldsymbol{u} \cdot(\boldsymbol{\nabla} \times \boldsymbol{u})}}{1+\sigma_{c}}, \\
\eta_{\mathrm{t}} & =\frac{\tau_{0} \overline{\boldsymbol{u}^{2}}}{3}\left[1-\frac{(q-1) \sigma_{c}}{2\left(1+\sigma_{c}\right)}\right],
\end{aligned}
$$




$$
\gamma=-\frac{1}{6} \nabla\left(\tau_{0} \overline{\boldsymbol{u}^{2}}\right)
$$

Equations (2.27) and (2.28) imply that for large magnetic Reynolds numbers, compressibility decrease both the $\alpha$ effect and turbulent magnetic diffusivity. Since $1<q<3$, the turbulent magnetic diffusivity is always positive for $\mathrm{Rm} \gg 1$, even for very large compressibility, $\sigma_{c} \gg 1$ (e.g., for irrotational flows). Note that for an incompressible flow the turbulent magnetic diffusivity is independent of $q$. On the other hand, compressibility of fluid flow does not affect the pumping velocity $\gamma$ of the mean magnetic field for $\mathrm{Rm} \gg 1$, similarly to the case of $\mathrm{Rm} \ll 1$.

In the framework of mean-field dynamo theory, the threshold for the generation of a mean magnetic field is formulated in terms of the dynamo number. In the case of an $\alpha^{2}$ dynamo, the dynamo number is given by $R_{\alpha}=\alpha L_{B} / \eta_{\mathrm{t}}$. Using (2.27) and (2.28), we find that, for a compressible flow, $R_{\alpha}$ is given by

$$
R_{\alpha}=R_{\alpha}^{(\mathrm{in})}\left(1+\frac{3-q}{2} \sigma_{c}\right)^{-1},
$$

where $R_{\alpha}^{(\text {in) }}$ applies to the corresponding incompressible flow. This implies that compressibility decreases the dynamo number.

\section{Turbulent transport of particles}

In this section we consider non-inertial particles or gaseous admixtures in a compressible fluid flow. Equation for the particle number density, $n^{(\mathrm{p})}(\boldsymbol{x}, t)$, reads (Chandrasekhar 1943; Akhiezer \& Peletminskii 1981):

$$
\frac{\partial n^{(\mathrm{p})}}{\partial t}+\boldsymbol{\nabla} \cdot\left(n^{(\mathrm{p})} \boldsymbol{U}-D \boldsymbol{\nabla} n^{(\mathrm{p})}\right)=0,
$$

where $D$ is the microphysical diffusivity describing Brownian diffusion of particles and $\boldsymbol{U}$ is a fluid velocity. Equation (3.1) implies conservation of the total number of particles in a closed volume. We consider one way coupling, i.e., we take into account the effect of turbulence on particle transport, but neglect the effect of particles on the turbulence. This corresponds to turbulent transport of a passive scalar.

To determine the turbulent flux of particles, we use a mean-field approach in which the number density of particles and fluid velocity are decomposed into mean and fluctuating parts, where the fluctuating parts have zero mean values. Averaging (3.1) over an ensemble, we arrive at an equation for the mean number density, $N(\boldsymbol{x}, t) \equiv \overline{n^{(\mathrm{p})}}$ :

$$
\frac{\partial N}{\partial t}+\nabla \cdot(\overline{n \boldsymbol{u}}-D \nabla N)=0
$$

where $\boldsymbol{F}=\overline{n(\boldsymbol{x}, t) \boldsymbol{u}(\boldsymbol{x}, t)}$ is the turbulent flux of particles and we consider the case of a zero mean fluid velocity, $\overline{\boldsymbol{U}}=0$.

To determine the turbulent flux of particles we use the equation for the fluctuations of the particle number density, $n(\boldsymbol{x}, t)=n^{(\mathrm{p})}-N$, which follows from (3.1) and (3.2):

$$
\frac{\partial n}{\partial t}+\nabla \cdot(n \boldsymbol{u}-\overline{n \boldsymbol{u}}-D \boldsymbol{\nabla} n)=-N \boldsymbol{\nabla} \cdot \boldsymbol{u}-(\boldsymbol{u} \cdot \boldsymbol{\nabla}) N,
$$

where $\mathcal{Q}=\boldsymbol{\nabla} \cdot(n \boldsymbol{u}-\overline{n \boldsymbol{u}})$ are nonlinear terms and $I=-N \boldsymbol{\nabla} \cdot \boldsymbol{u}-(\boldsymbol{u} \cdot \boldsymbol{\nabla}) N$ is the source term of particle number density fluctuations. The ratio of the nonlinear terms to the diffusion term is the Péclet number, that is estimated as $\mathrm{Pe}=u_{0} \ell_{0} / D$. In the next 
subsections we derive equations for the turbulent transport coefficients of particles for small and large Péclet numbers.

\subsection{Turbulent transport coefficients for $\mathrm{Pe} \ll 1$}

We derive an equation for the turbulent flux of particles,

$$
\overline{n(\boldsymbol{x}, t) u_{j}(\boldsymbol{x}, t)}=\int F_{j}(\boldsymbol{k}, \omega, \boldsymbol{R}, t) d \omega d \boldsymbol{k},
$$

for small Péclet numbers using a quasi-linear approach, where

$$
F_{j}(\boldsymbol{k}, \omega, \boldsymbol{R}, t)=\int \overline{n\left(\boldsymbol{k}_{1}, \omega_{1}\right) u_{j}\left(\boldsymbol{k}_{2}, \omega_{2}\right)} \exp [i \Omega t+i \boldsymbol{K} \cdot \boldsymbol{R}] d \Omega d \boldsymbol{K} .
$$

For brevity of notations we omit below the large-scale variables $t$ and $\boldsymbol{R}$ in $F_{j}(\boldsymbol{k}, \omega, \boldsymbol{R}, t)$ and $N(\boldsymbol{R}, t)$. We neglect the nonlinear term $\mathcal{Q}$, but keep the molecular diffusion term in (3.3). We rewrite (3.3) in Fourier space and find the solution of this equation (see (A20) in Appendix (A). Here we apply the same approach that was used in section 2.2 i.e., we assume that there is a separation of spatial and time scales, $\ell_{0} \ll L_{N}$ and $\tau_{0} \ll t_{N}$, where $L_{N}$ and $t_{N}$ are the characteristic spatial and time scales of the mean particle number density variations. We perform calculations presented in Appendix $\mathrm{A}$ so that equation for the turbulent flux of particles is given by

$$
\begin{aligned}
F_{j}= & -\int G_{D}\left[N\left(i k_{i} f_{i j}+\frac{1}{2} \nabla_{i} f_{i j}-D k^{2} G_{D} k_{i m} \nabla_{m} f_{i j}\right)+\frac{1}{2}\left(f_{m j}-k_{i} \frac{\partial f_{i j}}{\partial k_{m}}\right.\right. \\
& \left.\left.-2 D k^{2} G_{D} k_{i m} f_{i j}\right) \nabla_{m} N\right] d \boldsymbol{k} d \omega,
\end{aligned}
$$

where $G_{D} \equiv G_{D}(\boldsymbol{k}, \omega)=\left(D \boldsymbol{k}^{2}+i \omega\right)^{-1}$ and $f_{i j}=f_{i j}(\boldsymbol{k}, \omega, \boldsymbol{R}, t)$. Since we consider

one way coupling, the correlation function $f_{i j}$ in (3.6) should be replaced by $f_{i j}^{(0)}$ for the background random flow with zero turbulent flux of particles. Using the model of background random flow given by (2.12) with zero kinetic helicity, and integrating in $\omega$ and $\boldsymbol{k}$-space, we arrive at an equation for the turbulent flux of particles:

$$
\boldsymbol{F}=N \boldsymbol{V}^{\mathrm{eff}}-D_{\mathrm{t}} \boldsymbol{\nabla} N
$$

where for small Péclet numbers (Pe $\ll 1$ ), the turbulent diffusivity $D_{\mathrm{t}}$ and the effective pumping velocity $\boldsymbol{V}^{\mathrm{eff}}$ are given by

$$
\begin{aligned}
D_{\mathrm{t}} & =\frac{(q-1)}{3(q+1)} \tau_{0} \overline{\boldsymbol{u}^{2}}\left(1-\frac{2 \sigma_{c}}{1+\sigma_{c}}\right) \mathrm{Pe} \equiv \frac{(q-1)}{3(q+1)}\left(\frac{1-\sigma_{c}}{1+\sigma_{c}}\right) \mathrm{Pe}^{2} D, \\
\boldsymbol{V}^{\mathrm{eff}} & =\frac{(q-1)}{3(q+1)} \tau_{0} \overline{\boldsymbol{u}^{2}}\left[\frac{1}{\left(1+\sigma_{c}\right)} \boldsymbol{\nabla} \ln \bar{\rho}+\frac{3 \sigma_{c}}{2\left(1+\sigma_{c}\right)} \boldsymbol{\nabla} \ln \overline{\boldsymbol{u}^{2}}\right] \mathrm{Pe} .
\end{aligned}
$$

Since $\tau_{0} \mathrm{Pe}=\ell_{0}^{2} / D$, the turbulent transport coefficients given by (3.8) and (3.9) are determined only by the microphysical diffusion time scale, $\ell_{0}^{2} / D$. Remarkably, equation (3.8) for the turbulent diffusivity of passive scalars $D_{\mathrm{t}}$ coincides with (2.19) for the turbulent magnetic diffusivity $\eta_{\mathrm{t}}$ after replacing Pe by $\mathrm{Rm}$.

Equation (3.8) implies that for small Péclet numbers, compressibility effects decrease the turbulent diffusivity. However, the total (effective) diffusivity, $D+D_{\mathrm{t}}$, cannot be negative, because for $\mathrm{Pe} \ll 1$, the molecular diffusivity is much larger than the turbulent one, $D \gg\left|D_{\mathrm{t}}\right|$. This result is consistent with that of Rädler et al. (2011), where it has been demonstrated that the total mean-field diffusivity for passive scalar transport in irrotational flows is smaller than the molecular diffusivity (see section 4 for a detailed comparison). 
The physics related to different terms in (3.9) will be discussed in the next section. In the present study of turbulent transport of passive scalar and non-inertial particles, we consider nonhelical turbulence, because there is no effect of the kinetic helicity on the particle flux - at least in the system investigated here [without rotation, the large-scale shear, and neglecting effects $\left.\sim \mathrm{O}\left(\lambda^{2} \overline{\boldsymbol{u}^{2}}, \nabla^{2} \overline{\boldsymbol{u}^{2}}, \lambda_{i} \nabla_{j} \overline{\boldsymbol{u}^{2}}\right)\right]$.

By means of the equation of state for a perfect gas, $\nabla \ln \bar{p}=\nabla \ln \bar{\rho}+\nabla \ln \bar{T}$, we rewrite (3.9) for the effective pumping velocity as:

$$
\boldsymbol{V}^{\mathrm{eff}}=\frac{(q-1)}{3(q+1)} \tau_{0} \overline{\boldsymbol{u}^{2}}\left[\frac{1}{\left(1+\sigma_{c}\right)}(\boldsymbol{\nabla} \ln \bar{p}-\boldsymbol{\nabla} \ln \bar{T})+\frac{3 \sigma_{c}}{2\left(1+\sigma_{c}\right)} \boldsymbol{\nabla} \ln \overline{\boldsymbol{u}^{2}}\right] \mathrm{Pe},(3.1
$$

where $\bar{T}$ and $\bar{p}$ are the mean temperature and the mean pressure, respectively. Note that, since the density-temperature correlation $\overline{\rho^{\prime} \vartheta}$ is much smaller than $\bar{\rho} \bar{T}$ (Chassaing et al. 2013), the equation of state for perfect gas is also valid for the mean quantities, where $\rho^{\prime}$ and $\vartheta$ are fluctuations of the fluid density and temperature.

\subsection{Turbulent transport coefficients for $\mathrm{Pe} \gg 1$}

In this section we determine the turbulent flux of particles for large Péclet and Reynolds numbers. Similar to the study performed in section 2.3 , we consider fully developed turbulence, where the Strouhal number is of the order of unity and the turbulent correlation time is scale-dependent, so we apply the Fourier transformation only in $\boldsymbol{k}$ space. Using (3.3) for the fluctuations $n$ and the Navier-Stokes equation for the velocity $\boldsymbol{u}$ written in Fourier space, we derive an equation for the instantaneous two-point correlation function

$$
F_{j}(\boldsymbol{k})=\int \overline{n(\boldsymbol{k}+\boldsymbol{K} / 2) u_{j}(-\boldsymbol{k}+\boldsymbol{K} / 2)} \exp [i \boldsymbol{K} \cdot \boldsymbol{R}] d \boldsymbol{K} .
$$

For brevity of notations we omit the large-scale variables $t$ and $\boldsymbol{R}$ in the function $F_{j}(\boldsymbol{k}, \boldsymbol{R}, t)$ and the mean number density $N(\boldsymbol{R}, t)$. To derive an evolution equation for $F_{j}(\boldsymbol{k})$, we perform calculations presented in Appendix $\mathbf{C}$ which yield

$$
\frac{\partial F_{j}}{\partial t}=-N\left(i k_{i} f_{i j}+\frac{1}{2} \nabla_{i} f_{i j}\right)-\frac{1}{2}\left(f_{i j}-k_{m} \frac{\partial f_{m j}}{\partial k_{i}}\right) \nabla_{i} N+\hat{\mathcal{M}} F_{i}^{(\mathrm{III})}(\boldsymbol{k}),
$$

where

$$
\hat{\mathcal{M}} F_{j}^{(\mathrm{III})}(\boldsymbol{k})=\int\left[\overline{n\left(\boldsymbol{k}_{1}\right) \frac{\partial u_{j}\left(\boldsymbol{k}_{2}\right)}{\partial t}}-\overline{\mathcal{Q}\left(\boldsymbol{k}_{1}\right) u_{j}\left(\boldsymbol{k}_{2}\right)}\right] \exp [i \boldsymbol{K} \cdot \boldsymbol{R}] d \boldsymbol{K}
$$

are the third-order moment terms in $\boldsymbol{k}$ space appearing due to the nonlinear terms.

We use the spectral $\tau$ approximation (2.24), where functions with superscript (0) correspond to background turbulence with zero turbulent particle flux. Therefore, (2.24) reduces to $\hat{\mathcal{M}} F_{i}^{(\mathrm{III})}(\boldsymbol{k})=-F_{i}(\boldsymbol{k}) / \tau(k)$. We also assume that the characteristic time of variation of the second moment $F_{i}(\boldsymbol{k})$ is substantially larger than the correlation time $\tau(k)$ on all turbulence scales. Therefore, the particle flux is given by

$$
F_{j}(\boldsymbol{k})=-\tau(k)\left\{\left(i k_{i} f_{i j}^{(0)}+\frac{1}{2} \nabla_{i} f_{i j}^{(0)}\right) N+\left[f_{m j}^{(0)}+\frac{k}{2}\left(\frac{\mathrm{d} \ln \tau}{\mathrm{d} k}\right) k_{i m} f_{i j}^{(0)}\right] \nabla_{m} N\right\} .
$$

Using the model of background turbulence for $f_{i j}^{(0)}$ given by (2.25) with a zero kinetic helicity, and integrating (3.13) in $\boldsymbol{k}$-space, we obtain the turbulent flux of particles (3.7), where the turbulent diffusivity $D_{\mathrm{t}}$ and the effective pumping velocity $\boldsymbol{V}^{\text {eff }}$ of non-inertial 
particles for $\mathrm{Pe} \gg 1$ are given by

$$
\begin{aligned}
D_{\mathrm{t}} & =\frac{\tau_{0} \overline{\boldsymbol{u}^{2}}}{3}\left[1-\frac{(q-1) \sigma_{c}}{2\left(1+\sigma_{c}\right)}\right], \\
\boldsymbol{V}^{\mathrm{eff}} & =\frac{\tau_{0} \overline{\boldsymbol{u}^{2}}}{3}\left[\frac{1}{\left(1+\sigma_{c}\right)} \boldsymbol{\nabla} \ln \bar{\rho}+\frac{\sigma_{c}}{2\left(1+\sigma_{c}\right)} \nabla \ln \overline{\boldsymbol{u}^{2}}\right] .
\end{aligned}
$$

Equation (3.14) for the turbulent diffusivity of particles $D_{\mathrm{t}}$ coincides with (2.28) for the turbulent magnetic diffusivity $\eta_{\mathrm{t}}$ after replacing Pe by Rm. Equation (3.14) implies that for large Péclet numbers, compressibility decreases the turbulent diffusivity of particles. Since $1<q<3$, the turbulent diffusivity is always positive when $\mathrm{Pe} \gg 1$, even for very strong compressibility, $\sigma_{c} \gg 1$.

Equation (3.15) determines effective pumping velocity, $\boldsymbol{V}^{\mathrm{eff}}$, of passive scalars and non-inertial particles. The first term in (3.15), and likewise in (3.9) for $\mathrm{Pe} \ll 1$, describes pumping of passive scalars caused by density stratification. This effect for low Mach numbers has been studied theoretically using various analytical approaches (Elperin et al. 1995, 1996, 1997, 2000, 2001; Pandya \& Mashayek 2002; Reeks|2005; Amir et al. 2017). It has also been detected in the direct numerical simulations of Brandenburg et al. (2012b), where the density stratification was caused by gravity. Their result is reproduced in Fig. 10 and compared with the corresponding turbulent pumping velocities of mean magnetic field, which is close to zero. This demonstrates very clearly the different natures of pumping of particles and magnetic fields in one and the same simulation. Here, the relevant components of the effective pumping velocities of particles, $\boldsymbol{V}^{\mathrm{eff}}$, and magnetic field, $\gamma$, have been determined using the test-field method for axisymmetric turbulence described in Brandenburg et al. (2012b). The simulations have been carried out for about 200 turnover times, for $\mathrm{Pe}=\mathrm{Re}=u_{\mathrm{rms}} / \nu k_{\mathrm{f}}=22$ and $k_{\mathrm{f}} / k_{1}=5$, where $k_{1}$ is the wavenumber based on the size of the computational domain and $k_{\mathrm{f}}$ is the forcing scale of turbulence. Error bars have been determined using any one third of the full time series of the instantaneous, but spatially averaged mean values of $\boldsymbol{V}^{\mathrm{eff}}$ and $\boldsymbol{\gamma}$. Within error bars, the result for $\gamma$ is not quite compatible with zero, but this is entirely explicable as a consequence of a small upward increase in the rms velocity with height and thus a small positive gradient. Also, the deviation of the theoretical from the numerical results for larger $\left(k_{\mathrm{f}} H\right)^{-1}$ is caused by the fact that the parameter $\ell_{0} / H$ is no longer very small, as was assumed in the theory. The effective pumping velocity of particles $\boldsymbol{V}^{\text {eff }}$ for arbitrary stratifications has been determined analytically by Amir et al. (2017). They have shown that there is a quenching of the pumping velocity caused by the strong stratification. This tendency is already seen in Fig. 1 (dotted line) for larger density stratifications.

Let us discuss the physics related to the first term in the right hand side of (3.15) caused by the fluid density stratification, considering density stratified homogeneous turbulence. Substituting (3.7) into (3.2), we obtain the equation for the mean number density, $N$ :

$$
\frac{\partial N}{\partial t}+\boldsymbol{\nabla} \cdot\left[N \boldsymbol{V}^{\mathrm{eff}}-\left(D+D_{\mathrm{t}}\right) \boldsymbol{\nabla} N\right]=0 .
$$

The steady-state solution of Eqs. (3.16) for the mean number density of non-inertial particles is given by $N / N_{0}=\left[\bar{\rho} / \bar{\rho}_{0}\right]^{D_{\mathrm{t}} /\left(D+D_{\mathrm{t}}\right)}$, where subscripts 0 represent the values in the region with homogeneous fluid density. Here we used Eqs. (3.8) and (3.9) or (3.14) and (3.15) for homogeneous turbulence. This solution implies that small particles are accumulated in the vicinity of the maximum of the fluid density, because the non-diffusive flux of particles, $N \boldsymbol{V}^{\text {eff }}$, is directed toward the maximum of the fluid density.

The accumulation of non-inertial particles in the vicinity of the maximum of the mean 


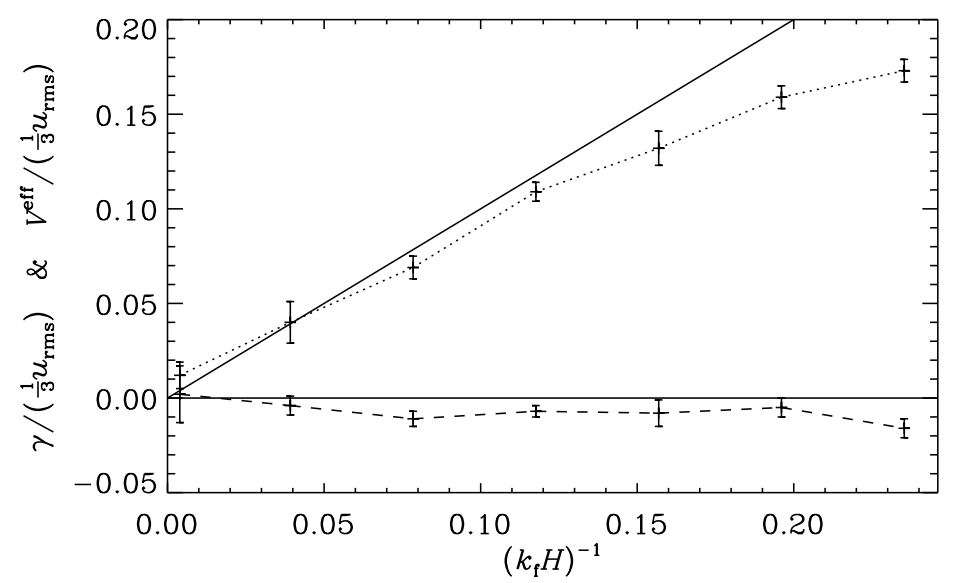

Figure 1. Dependence of $V^{\text {eff }}$ (dotted line) and $\gamma$ (dashed line) (here in the $z$ direction) as a function of the inverse scale height $H$, normalized by the wavenumber $k_{\mathrm{f}}$ of the energy-carrying eddies. The turbulent pumping velocity $\gamma$ caused by the nonuniformity of the turbulent rms velocity (solid line) in a nonstratified turbulence. Here the symbol $H$ is used either for the density scale height, $H=\lambda^{-1}$, or for the characteristic scale of the nonuniformity of the turbulent rms velocity, $H^{-1}=\nabla u_{\mathrm{rms}} / u_{\mathrm{rms}}$.

fluid density can be explained as follows (Elperin et al. 1995, 1997; Haugen et al. 2012). Let us assume that the mean fluid density is inhomogeneous along the $x$ axis, and the mean density $\bar{\rho}_{2}$ at point 2 is larger than the mean density $\bar{\rho}_{1}$ at point 1 . Consider two small control volumes "a" and "b" located between these two points, and let the direction of the local turbulent velocity in volume "a" at some instant be the same as the direction of the mean fluid density gradient $\nabla \bar{\rho}$ (e.g., along the $x$ axis toward point 2 ). Let the local turbulent velocity in volume "b" at this instant be directed opposite to the mean fluid density gradient (i.e., toward point 1). In a fluid flow with an imposed mean fluid density gradient, one of the sources of particle number density fluctuations, $n \propto-\tau_{0} N(\boldsymbol{\nabla} \cdot \boldsymbol{u})$, is caused by a non-zero $\boldsymbol{\nabla} \cdot \boldsymbol{u} \approx-\boldsymbol{u} \cdot \boldsymbol{\nabla} \ln \bar{\rho} \neq 0$ [see the first term on the right hand side of Eq. [3.3)]. Since fluctuations of the fluid velocity $u_{x}$ are positive in volume "a" and negative in volume "b", $\boldsymbol{\nabla} \cdot \boldsymbol{u}<0$ in volume "a", and $\boldsymbol{\nabla} \cdot \boldsymbol{u}>0$ in volume "b". Therefore, the fluctuations of the particle number density $n \propto-\tau_{0} N(\boldsymbol{\nabla} \cdot \boldsymbol{u})$ are positive in volume "a" and negative in volume "b". However, the flux of particles $n u_{x}$ is positive in volume "a" (i.e., it is directed toward point 2), and it is also positive in volume "b" (because both fluctuations of fluid velocity and number density of particles are negative in volume "b"). Therefore, the mean flux of particles $\overline{n \boldsymbol{u}}$ is directed, as is the mean fluid density gradient $\nabla \bar{\rho}$, toward point 2 . This forms large-scale heterogeneous structures of non-inertial particles in regions with a mean fluid density maximum.

Indirect manifestations of the pumping effect caused by the density stratification through an observed increase of small-scale clustering of particles can be identified in the direct numerical simulations of van Aartrijk \& Clercx (2008), who studied the evolution of inertial particles in stably stratified turbulence. This pumping effect increases the small-scale clustering of inertial particles by forming inhomogeneous distributions of the mean particle number density. Note also that tangling of the mean particle number density, $N$, by compressible velocity fluctuations (described by the source term $-N \operatorname{div} \boldsymbol{u}$ in (3.3)), can increase the level of particle number density fluctuations, $n$, when the source 
term $-N \operatorname{div} \boldsymbol{u}>0$. In this case $\partial n / \partial t>0$, see (3.3). This can amplify the particle clustering (Eidelman et al. 2010; Elperin et al. 2013).

Using the equation of state for a perfect gas, we rewrite (3.15) for the pumping effective velocity in the form

$$
\boldsymbol{V}^{\mathrm{eff}}=\frac{\tau_{0} \overline{\boldsymbol{u}^{2}}}{3}\left[\frac{1}{\left(1+\sigma_{c}\right)}(\nabla \ln \bar{p}-\nabla \ln \bar{T})+\frac{\sigma_{c}}{2\left(1+\sigma_{c}\right)} \nabla \ln \overline{\boldsymbol{u}^{2}}\right] .
$$

The first term in the right hand side of (3.17) describes turbulent barodiffusion (Elperin et al. 1997), while the second term characterizes the phenomenon of turbulent thermal diffusion (Elperin et al. 1996, 2000; Amir et al. 2017). The last term in (3.17), $\propto \sigma_{c} \boldsymbol{\nabla} \overline{\boldsymbol{u}^{2}}$, describes the compressible turbophoresis for noninertial particles.

The classical turbophoresis (Caporaloni et al. 1975; Reeks 1983, 1992; Elperin et al. 1998a; Mitra et al. 2018) exists only for inertial particles and causes an additional mean particle velocity that is proportional to $-\tau_{s} \boldsymbol{\nabla} \overline{\boldsymbol{u}^{2}}$, where $\tau_{s}=m_{p} / 6 \pi \rho \nu a_{p}$ is the particle Stokes time, i.e., the characteristic time of coupling between small spherical particles (of radius $a_{p}$ and mass $m_{p}$ ) and surrounding fluid. To illustrate the classical turbophoresis effect, let us consider small inertial particles in an isotropic incompressible turbulence and use equation of motion for a particle: $d \boldsymbol{v}^{(\mathrm{p})} / d t=-\left(\boldsymbol{v}^{(\mathrm{p})}-\boldsymbol{u}\right) / \tau_{s}$, where $\boldsymbol{v}^{(\mathrm{p})}$ is the particle velocity. This equation represents a balance of particle inertia with the fluid drag force produced by the motion of the particle relative to the surrounding fluid. Solution of this equation for small Stokes time is given by: $\boldsymbol{v}^{(\mathrm{p})}=\boldsymbol{u}-\tau_{s}[\partial \boldsymbol{u} / \partial t+(\boldsymbol{u} \cdot \boldsymbol{\nabla}) \boldsymbol{u}]+\mathrm{O}\left(\tau_{s}^{2}\right)$ (Maxey 1987). Averaging this equation over statistics of fluid velocity fluctuations, we determine the mean particle velocity: $\bar{v}_{j}^{(\mathrm{p})}=-\tau_{s} \nabla_{j} \overline{u_{i} u_{j}}$. We take into account that for isotropic turbulence $\overline{u_{i} u_{j}}=(1 / 3) \overline{\boldsymbol{u}^{2}} \delta_{i j}$. Therefore, the mean particle velocity is $\overline{\boldsymbol{v}}^{(\mathrm{p})}=$ $-\left(\tau_{s} / 3\right) \nabla \overline{\boldsymbol{u}^{2}}$ (for details, see Elperin et al. 1998a). This turbophoresis effect results in the formation of large-scale clusters of inertial particles at local minima of the mean-squared turbulent velocity. Note that the compressible turbophoresis for noninertial particles originates from the turbulent particle flux $\overline{n \boldsymbol{u}}$, i.e., it describes a collective statistical phenomenon, while classical turbophoresis is not related to the correlations between velocity and number density fluctuations and is obtained from the expression for the mean velocity of inertial particles.

In the case of rapidly rotating turbulence, another pumping effect of inertial particles arises which can accumulate particles (Hodgson \& Brandenburg 1998; Elperin et al. 1998b). This effect can be related to the combined action of particle inertia and non-zero mean kinetic helicity in rotating turbulence.

\section{Comparison with Krause \& Rädler (1980) and Rädler et al. (2011)}

In this section we compare our results with those obtained previously by Krause \& Rädler (1980) and Rädler et al. (2011). Let us consider non-stratified, isotropic, homogeneous and non-helical random flow. In this case (2.12) reads:

$$
f_{i j}^{(0)}(\boldsymbol{k}, \omega, \boldsymbol{R})=\frac{\overline{\boldsymbol{u}^{2}} \Phi_{u}(\omega) E(k)}{8 \pi k^{2}\left(1+\sigma_{c}\right)}\left[\delta_{i j}-k_{i j}+2 \sigma_{c} k_{i j}\right],
$$

where the first two terms, $\delta_{i j}-k_{i j}$, in the squared brackets describes the vortical part of the turbulent flow, while the last term, $2 \sigma_{c} k_{i j}$ determines the potential part of the flow. Representing the velocity fluctuations as the sum of vortical and potential parts,

$$
\boldsymbol{u}=\nabla \times \psi+\nabla \phi
$$


we can construct the correlation function, $f_{i j}^{(0)}(\boldsymbol{k})=\overline{u_{i}(\boldsymbol{k}) u_{j}(-\boldsymbol{k})}$, of the velocity field in $k$ space for non-stratified, isotropic, homogeneous and non-helical random background flow as

$$
f_{i j}^{(0)}(\boldsymbol{k})=\frac{\overline{\boldsymbol{u}^{2}} E(k)}{8 \pi k^{2}\left(1+\overline{\phi^{2}} / \overline{\boldsymbol{\psi}^{2}}\right)}\left(\delta_{i j}-k_{i j}+\frac{2 \overline{\phi^{2}}}{\overline{\boldsymbol{\psi}^{2}}} k_{i j}\right),
$$

where $\boldsymbol{\nabla} \cdot \boldsymbol{\psi}=0$, and we also assumed that compressible and incompressible parts of the random velocity field have the same spectra, $E(k)$. It follows from (4.1) and (4.3) that the degree of compressibility is $\sigma_{c}=\overline{\phi^{2}} / \overline{\psi^{2}}$. For simplicity, we consider here the instantaneous correlation function.

To derive (4.3), we rewrite (4.2) in $\boldsymbol{k}$ space: $u_{i}(\boldsymbol{k})=i k_{m} \varepsilon_{i m n} \psi_{n}(\boldsymbol{k})+i k_{i} \phi(\boldsymbol{k})$, and assume that the vortical and potential parts are not correlated. This allows us to obtain the following expression for $f_{i j}^{(0)}(\boldsymbol{k})$ :

$$
f_{i j}^{(0)}(\boldsymbol{k})=k^{2}\left[\varepsilon_{i m n} \varepsilon_{j p q} k_{m p} \overline{\psi_{n}(\boldsymbol{k}) \psi_{q}(-\boldsymbol{k})}+k_{i j} \overline{\phi(\boldsymbol{k}) \phi(-\boldsymbol{k})}\right],
$$

where

$$
\overline{\psi^{2}}=\int \overline{\psi_{n}(\boldsymbol{k}) \psi_{n}(-\boldsymbol{k})} d \boldsymbol{k}, \quad \overline{\phi^{2}}=\int \overline{\phi(\boldsymbol{k}) \phi(-\boldsymbol{k})} d \boldsymbol{k} .
$$

Taking into account that for the vortical field $\boldsymbol{\nabla} \cdot \boldsymbol{\psi}=0$, the correlation function is of the form $\overline{\psi_{i}(\boldsymbol{k}) \psi_{j}(-\boldsymbol{k})} \propto \delta_{i j}-k_{i j}$, and using (4.5) we obtain

$$
\begin{aligned}
& \overline{\psi_{i}(\boldsymbol{k}) \psi_{j}(-\boldsymbol{k})}=\frac{\overline{\boldsymbol{u}^{2}} E(k)}{8 \pi k^{4}\left(1+\overline{\phi^{2}} / \overline{\boldsymbol{\psi}^{2}}\right)}\left(\delta_{i j}-k_{i j}\right), \\
& \overline{\phi(\boldsymbol{k}) \phi(-\boldsymbol{k})}=\frac{\overline{\boldsymbol{u}^{2}} E(k)}{4 \pi k^{4}\left(1+\overline{\boldsymbol{\psi}^{2}} / \overline{\phi^{2}}\right)} .
\end{aligned}
$$

Substituting (4.6)-(4.7) into (4.4), we arrive at (4.3).

\subsection{Small magnetic Reynolds numbers}

Substituting (4.3) into (2.16) and using (2.11), we find that the turbulent magnetic diffusivity for small magnetic Reynolds numbers reads:

$$
\eta_{\mathrm{t}}=\frac{1}{3 \eta}\left(\overline{\psi^{2}}-\overline{\phi^{2}}\right) \text {. }
$$

This result agrees with that of Krause \& Rädler (1980). Substituting (4.3) into (3.6), we find that the turbulent diffusion coefficient for passive scalars at small Péclet numbers is given by:

$$
D_{\mathrm{t}}=\frac{1}{3 D}\left(\overline{\psi^{2}}-\overline{\phi^{2}}\right) .
$$

This result also agrees with that of Rädler et al. (2011).

\subsection{Large magnetic Reynolds numbers}

In the case of large magnetic Reynolds numbers, substituting (4.3) into (2.26), we find for the turbulent magnetic diffusivity

$$
\eta_{\mathrm{t}}=\frac{\tau_{0} \overline{\boldsymbol{u}^{2}}}{3}\left[1-\frac{(q-1)}{2}\left(\frac{\overline{\phi^{2}}}{\overline{\psi^{2}}+\overline{\phi^{2}}}\right)\right] .
$$


This result is in good agreement with (2.28) for $\eta_{\mathrm{t}}$ and with (3.14) for $D_{\mathrm{t}}$.

\section{Conclusions}

A mean-field theory for the compressibility effects in the mean electromotive force in turbulent magnetohydrodynamics and in the turbulent flux of passive scalars or particles has been developed. This study is based on the quasi-linear approach applied for small magnetic Reynolds and Péclet numbers, and on the spectral $\tau$-approach for large fluid $(\mathrm{Re})$ and magnetic $(\mathrm{Rm})$ Reynolds numbers and large Péclet numbers (Pe). Compressibility is found to cause a depletion of the $\alpha$ effect and turbulent magnetic diffusion for small and large Rm. It also decreases the turbulent diffusivity of passive scalars for small and large Pe. Indeed, the expressions for the turbulent magnetic diffusivity and the turbulent diffusivity of passive scalars coincide after replacing $\mathrm{Rm}$ by $\mathrm{Pe}$, and vise versa. Compressibility does not change the effective pumping velocity of the magnetic field for $\mathrm{Rm} \gg 1$, but decreases it for $\mathrm{Rm} \ll 1$. In addition, compressibility causes a pumping of particles in regions with higher turbulent intensity for small and large Péclet numbers. This new effect is interpreted in terms of compressible turbophoresis for noninertial particles. This effect is completely different from classical turbophoresis, which only affects inertial particles and results in pumping of inertial particles to regions of lower turbulent intensity. Compressible turbophoresis for noninertial particles is a collective statistical phenomenon originating from the turbulent particle flux. On the other hand, classical turbophoresis originates directly from the expression for the mean velocity of inertial particles. For small Mach numbers, compressibility effects are only determined by the density stratification through the $\lambda$ terms in (2.12) and (2.25). We find that the density stratification does not affect the magnetic field, although it causes a turbulent pumping of particles to regions of maximum mean fluid density.

The mean-field theory developed for compressibility effects in turbulent magnetohydrodynamics and turbulent transport of passive scalars and particles needs to be verified in direct numerical simulations and large-eddy simulations using the test-field and testscalar methods (see, e.g., Schrinner et al. 2005, 2007; Brandenburg et al. 2008, 2012b), which is the subject of a separate study. Furthermore, in view of astrophysical and geophysical applications, it is important to consider the additional effects of rotation, turbulent convection, and the presence of stably stratified turbulence with large-scale temperature gradients (see, e.g., Kleeorin \& Rogachevskii 2003; Rogachevskii \& Kleeorin 2006, 2007; Brandenburg et al. 2012b).

We thank Matthias Rheinhardt for his many questions and suggestions which have significantly improved the clarity of the presentation. This work was supported in part by the Research Council of Norway under the FRINATEK (grant No. 231444), the Israel Science Foundation governed by the Israeli Academy of Sciences (grant No. 1210/15), the National Science Foundation under grants No. NSF PHY-1748958 and AAG-1615100, and the University of Colorado through its support of the George Ellery Hale visiting faculty appointment. I.R. acknowledges the hospitality of Nordita, the Laboratory for Atmospheric and Space Physics of the University of Colorado, the Kavli Institute for Theoretical Physics in Santa Barbara and the École Polytechnique Fédérale de Lausanne. 
Appendix A. Derivation of (2.11) for $\mathrm{Rm} \ll 1$, and (3.6) for $\mathrm{Pe} \ll 1$

In the limit of small magnetic Reynolds number, we neglect in 2.5) nonlinear terms, but keep molecular dissipative terms of the magnetic fluctuations. The solution of (2.5) reads:

$$
b_{i}(\boldsymbol{k}, \omega)=i k_{p} G_{\eta}(\boldsymbol{k}, \omega)\left[\int \bar{B}_{p}(\boldsymbol{Q}) u_{i}(\boldsymbol{k}-\boldsymbol{Q}, \omega) d \boldsymbol{Q}-\int \bar{B}_{i}(\boldsymbol{Q}) u_{p}(\boldsymbol{k}-\boldsymbol{Q}, \omega) d \boldsymbol{Q}\right],
$$

where $G_{\eta}(\boldsymbol{k}, \omega)=\left(\eta \boldsymbol{k}^{2}+i \omega\right)^{-1}$. To derive A 1), we consider the source term in the induction equation for the magnetic fluctuations, $(\overline{\boldsymbol{B}} \cdot \boldsymbol{\nabla}) \boldsymbol{u}$, which can be rewritten using Fourier transformation as

$$
(\overline{\boldsymbol{B}} \cdot \boldsymbol{\nabla}) u_{i}=\int \bar{B}_{p}(\boldsymbol{Q}) \exp (i \boldsymbol{Q} \cdot \boldsymbol{x}) d \boldsymbol{Q} \int i k_{p}^{\prime} u_{i}\left(\boldsymbol{k}^{\prime}\right) \exp \left(i \boldsymbol{k}^{\prime} \cdot \boldsymbol{x}\right) d \boldsymbol{k}^{\prime} .
$$

For brevity of notation we omit hereafter the arguments $t$ and $\boldsymbol{x}$ in the functions $\overline{\boldsymbol{B}}(\boldsymbol{x}, t)$ and $\boldsymbol{u}(\boldsymbol{x}, t)$, and also the variable $t$ in the functions $\bar{B}_{p}(\boldsymbol{Q}, t)$ and $u_{i}(\boldsymbol{k}, t)$. We introduce a new variable $\boldsymbol{k}=\boldsymbol{k}^{\prime}+\boldsymbol{Q}$ and take into account that $Q_{p} \bar{B}_{p}(\boldsymbol{Q})=0$, which yields

$$
\begin{aligned}
(\overline{\boldsymbol{B}} \cdot \boldsymbol{\nabla}) u_{i} & =\int\left(i k_{p} \int \bar{B}_{p}(\boldsymbol{Q}) u_{i}(\boldsymbol{k}-\boldsymbol{Q}) d \boldsymbol{Q}\right) \exp (i \boldsymbol{k} \cdot \boldsymbol{x}) d \boldsymbol{k} \\
& \equiv \int\left[(\overline{\boldsymbol{B}} \cdot \boldsymbol{\nabla}) u_{i}\right]_{\boldsymbol{k}} \exp (i \boldsymbol{k} \cdot \boldsymbol{x}) d \boldsymbol{k},
\end{aligned}
$$

where $[\ldots]_{k}$ denotes the Fourier transform. Therefore,

$$
\left[(\overline{\boldsymbol{B}} \cdot \boldsymbol{\nabla}) u_{i}\right]_{\boldsymbol{k}}=i k_{p} \int \bar{B}_{p}(\boldsymbol{Q}) u_{i}(\boldsymbol{k}-\boldsymbol{Q}) d \boldsymbol{Q} .
$$

Similar derivations are used for the other terms in the induction equation:

$$
\begin{aligned}
{\left[(\boldsymbol{u} \cdot \boldsymbol{\nabla}) \bar{B}_{i}\right]_{\boldsymbol{k}} } & =i \int Q_{p} \bar{B}_{i}(\boldsymbol{Q}) u_{p}(\boldsymbol{k}-\boldsymbol{Q}) d \boldsymbol{Q}, \\
{\left[\bar{B}_{i}(\boldsymbol{\nabla} \cdot \boldsymbol{u})\right]_{\boldsymbol{k}} } & =i \int\left(k_{p}-Q_{p}\right) \bar{B}_{i}(\boldsymbol{Q}) u_{p}(\boldsymbol{k}-\boldsymbol{Q}) d \boldsymbol{Q},
\end{aligned}
$$

which yield (A 1).

To derive (2.11), we use (2.7) and (A 1) which yield

$$
g_{i j}(\boldsymbol{k}, \boldsymbol{R})=J_{i j}^{(2)}(\boldsymbol{k}, \boldsymbol{R})-J_{i j}^{(1)}(\boldsymbol{k}, \boldsymbol{R}),
$$

where

$$
\begin{aligned}
J_{i j}^{(1)}(\boldsymbol{k}, \boldsymbol{R})= & i \int\left(k_{p}+K_{p} / 2\right) G_{\eta}(\boldsymbol{k}+\boldsymbol{K} / 2) \overline{u_{p}(\boldsymbol{k}+\boldsymbol{K} / 2-\boldsymbol{Q}) u_{j}(-\boldsymbol{k}+\boldsymbol{K} / 2)} \\
& \times \bar{B}_{i}(\boldsymbol{Q}) \exp (i \boldsymbol{K} \cdot \boldsymbol{R}) d \boldsymbol{K} d \boldsymbol{Q} \\
J_{i j}^{(2)}(\boldsymbol{k}, \boldsymbol{R})= & i \int\left(k_{p}+K_{p} / 2\right) G_{\eta}(\boldsymbol{k}+\boldsymbol{K} / 2) \overline{u_{i}(\boldsymbol{k}+\boldsymbol{K} / 2-\boldsymbol{Q}) u_{j}(-\boldsymbol{k}+\boldsymbol{K} / 2)} \\
& \times \bar{B}_{p}(\boldsymbol{Q}) \exp (i \boldsymbol{K} \cdot \boldsymbol{R}) d \boldsymbol{K} d \boldsymbol{Q},
\end{aligned}
$$

and the functions $J_{i j}^{(1)}, J_{i j}^{(2)}, G_{\eta}$ and $u_{i}$ depend also on $\omega$. To simplify the notation, we do not show this dependence in the following calculations. First we determine the function $J_{i j}^{(1)}$, using new variables:

$$
\tilde{\boldsymbol{k}}_{1}=\boldsymbol{k}+\boldsymbol{K} / 2-\boldsymbol{Q}, \quad \tilde{\boldsymbol{k}}_{2}=-\boldsymbol{k}+\boldsymbol{K} / 2,
$$




$$
\tilde{\boldsymbol{k}}=\left(\tilde{\boldsymbol{k}}_{1}-\tilde{\boldsymbol{k}}_{2}\right) / 2=\boldsymbol{k}-\boldsymbol{Q} / 2, \tilde{\boldsymbol{K}}=\tilde{\boldsymbol{k}}_{1}+\tilde{\boldsymbol{k}}_{2}=\boldsymbol{K}-\boldsymbol{Q} .
$$

Therefore,

$$
\begin{aligned}
J_{i j}^{(1)}(\boldsymbol{k}, \boldsymbol{R})= & i \int\left(k_{p}+K_{p} / 2\right) f_{p j}(\boldsymbol{k}-\boldsymbol{Q} / 2, \boldsymbol{K}-\boldsymbol{Q}) G_{\eta}(\boldsymbol{k}+\boldsymbol{K} / 2) \bar{B}_{i}(\boldsymbol{Q}) \\
& \times \exp (i \boldsymbol{K} \cdot \boldsymbol{R}) d \boldsymbol{K} d \boldsymbol{Q} .
\end{aligned}
$$

Since $|\boldsymbol{Q}| \ll|\boldsymbol{k}|$ and $|\boldsymbol{K}| \ll|\boldsymbol{k}|$, we use the Taylor expansion

$$
\begin{aligned}
& f_{p j}(\boldsymbol{k}-\boldsymbol{Q} / 2, \boldsymbol{K}-\boldsymbol{Q})=f_{p j}(\boldsymbol{k}, \boldsymbol{K}-\boldsymbol{Q})-\frac{1}{2} \frac{\partial f_{p j}}{\partial k_{s}} Q_{s}+O\left(\boldsymbol{Q}^{2}\right), \\
& G_{\eta}(\boldsymbol{k}+\boldsymbol{K} / 2)=G_{\eta}(\boldsymbol{k})\left[1-\eta(\boldsymbol{k} \cdot \boldsymbol{K}) G_{\eta}(\boldsymbol{k})\right]+O\left(\boldsymbol{K}^{2}\right) .
\end{aligned}
$$

Substituting (A 12) and A 13 into (A 11), we get

$$
\begin{aligned}
& J_{i j}^{(1)}(\boldsymbol{k}, \boldsymbol{R})=i G_{\eta}(\boldsymbol{k}) \int\left[\left(k_{p}-\eta G_{\eta}(\boldsymbol{k}) k_{p} k_{s} K_{s}+\frac{1}{2} K_{p}\right)\left(\int f_{p j}(\boldsymbol{k}, \boldsymbol{K}-\boldsymbol{Q}) \bar{B}_{i}(\boldsymbol{Q}) d \boldsymbol{Q}\right)\right. \\
& \left.-\frac{1}{2} k_{p}\left(\int Q_{s} \bar{B}_{i}(\boldsymbol{Q}) \frac{\partial}{\partial k_{s}} f_{p j}(\boldsymbol{k}, \boldsymbol{K}-\boldsymbol{Q}) d \boldsymbol{Q}\right)\right] \exp (i \boldsymbol{K} \cdot \boldsymbol{R}) d \boldsymbol{K}+O\left(\boldsymbol{K}^{2}, \boldsymbol{Q}^{2}, K_{p} Q_{s}\right) .
\end{aligned}
$$

We use the following identity:

$$
\nabla_{p}\left[f_{p j}(\boldsymbol{k}, \boldsymbol{R}) \bar{B}_{i}(\boldsymbol{R})\right]=\int i K_{p}\left[f_{p j}(\boldsymbol{k}, \boldsymbol{K}) \bar{B}_{i}(\boldsymbol{K})\right]_{\boldsymbol{K}} \exp (i \boldsymbol{K} \cdot \boldsymbol{R}) d \boldsymbol{K},
$$

where

$$
\left[f_{p j}(\boldsymbol{k}, \boldsymbol{K}) \bar{B}_{i}(\boldsymbol{K})\right]_{\boldsymbol{K}}=\int f_{p j}(\boldsymbol{k}, \boldsymbol{K}-\boldsymbol{Q}) \bar{B}_{i}(\boldsymbol{Q}) d \boldsymbol{Q} .
$$

Similarly,

$$
f_{p j}(\boldsymbol{k}, \boldsymbol{R}) \nabla_{p} \bar{B}_{i}(\boldsymbol{R})=\int\left[\int f_{p j}(\boldsymbol{k}, \boldsymbol{K}-\boldsymbol{Q}) i Q_{p} \bar{B}_{i}(\boldsymbol{Q}) d \boldsymbol{Q}\right]_{\boldsymbol{K}} \exp (i \boldsymbol{K} \cdot \boldsymbol{R}) d \boldsymbol{K} .(\mathrm{A}
$$

Therefore, (A14)- A 17) yield

$$
\begin{aligned}
J_{i j}^{(1)}(\boldsymbol{k}, \boldsymbol{R})= & G_{\eta}\left\{\left[i k_{p}\left(1+i \eta G_{\eta} k_{s} \nabla_{s}\right)\left(\bar{B}_{i} f_{p j}\right)+\bar{B}_{i} \frac{1}{2} \nabla_{p} f_{p j}\right]\right. \\
& \left.+\frac{1}{2}\left(f_{p j}-k_{m} \frac{\partial f_{m j}}{\partial k_{p}}\right)\left(\nabla_{p} \bar{B}_{i}\right)\right\}+O\left(\boldsymbol{\nabla}^{2}\right) .
\end{aligned}
$$

A similar derivation is also performed for $J_{i j}^{(2)}$, which yields

$$
\begin{aligned}
J_{i j}^{(2)}(\boldsymbol{k}, \boldsymbol{R})= & G_{\eta}\left\{\bar{B}_{p}\left[i k_{p} f_{i j}+\frac{1}{2} \nabla_{p} f_{i j}-\eta k^{2} G_{\eta} k_{s p} \nabla_{s} f_{i j}\right]\right. \\
& \left.-\left(\nabla_{s} \bar{B}_{p}\right)\left[\frac{1}{2} k_{p} \frac{\partial f_{i j}}{\partial k_{s}}+\eta k^{2} G_{\eta} k_{s p} f_{i j}\right]\right\}+O\left(\nabla^{2}\right) .
\end{aligned}
$$

Therefore, (A 18) and (A 19) yield (2.11).

To determine the turbulent flux of particles for small Péclet numbers, we rewrite (3.3) in Fourier space using an equation similar to (A 4), and find the solution of (3.3) as

$$
n(\boldsymbol{k}, \omega)=-G_{D}(\boldsymbol{k}, \omega) i k_{p} \int N(\boldsymbol{Q}) u_{p}(\boldsymbol{k}-\boldsymbol{Q}, \omega) d \boldsymbol{Q},
$$


where $G_{D}(\boldsymbol{k}, \omega)=\left(D \boldsymbol{k}^{2}+i \omega\right)^{-1}$. The function $F_{j}(\boldsymbol{k}, \boldsymbol{R})$, defined by (3.5), is

$$
\begin{aligned}
F_{j}(\boldsymbol{k}, \boldsymbol{R})= & -i \int\left(k_{p}+K_{p} / 2\right) \overline{u_{p}(\boldsymbol{k}+\boldsymbol{K} / 2-\boldsymbol{Q}) u_{j}(-\boldsymbol{k}+\boldsymbol{K} / 2)} \\
& \times G_{D}(\boldsymbol{k}+\boldsymbol{K} / 2) N(\boldsymbol{Q}) \exp (i \boldsymbol{K} \cdot \boldsymbol{R}) d \boldsymbol{K} d \boldsymbol{Q},
\end{aligned}
$$

where the functions $F_{j}, G_{D}$ and $u_{i}$ depend also on $\omega$, and $N$ depend on $t$ as well. To simplify the notation, we do not show this dependence here. We perform calculations similar to those in (A 8)- A 18). In particular, after the Taylor expansion for $|\boldsymbol{Q}| \ll|\boldsymbol{k}|$ and $|\boldsymbol{K}| \ll|\boldsymbol{k}|$, we arrive at expression (3.6) for the turbulent flux of particles in Fourier space for small Péclet numbers.

\section{Appendix B. Derivation of $(2.20)$ for $\mathbf{R m} \ll 1$}

The effective pumping velocity $\gamma$ of the large-scale magnetic field is independent of $\boldsymbol{\lambda}$ for $\mathrm{Rm} \ll 1$. Indeed, the contribution of $\boldsymbol{\lambda}$ to the effective pumping velocity $\gamma$ can only arise from the term

$$
g_{i j}^{(1)}(\boldsymbol{k}, \omega)=i k_{p} G_{\eta}(k, \omega)\left[\bar{B}_{p} f_{i j}(\boldsymbol{k}, \omega)-\bar{B}_{i} f_{p j}(\boldsymbol{k}, \omega)\right] .
$$

Integration over solid angles in $\boldsymbol{k}$ space in $g_{i j}^{(1)}=\int g_{i j}^{(1)}(\boldsymbol{k}, \omega) d \boldsymbol{k} d \omega$ yields

$g_{i j}^{(1)}=-\frac{\int d k E(k) \int d \omega \Phi_{u}(\omega) G_{\eta}(k, \omega)}{6\left(1+\sigma_{c}\right)}\left[\bar{B}_{i} \lambda_{j}+\bar{B}_{j} \lambda_{i}-\left(\sigma_{c}+\frac{1}{2}\right)\left(\bar{B}_{i} \nabla_{j}+\bar{B}_{j} \nabla_{i}\right) \overline{\boldsymbol{u}^{2}}\right]$,

which is a symmetric tensor with respect to the indexes $i$ and $j$. Therefore, it cannot contribute to the mean electromotive force nor to $\gamma$. For integration over solid angles in $\boldsymbol{k}$ space in the expression for $g_{i j}^{(1)}$, we take into account that $\int k_{i j} \sin \theta d \theta d \varphi=(4 \pi / 3) \delta_{i j}$, where we use spherical coordinates $(k, \theta, \varphi)$ in $\boldsymbol{k}$ space.

The remaining contribution, which is proportional to the mean magnetic field, is

$$
g_{i j}^{(2)}(\boldsymbol{k}, \omega)=G_{\eta}\left[\bar{B}_{p}\left(\frac{1}{2} \nabla_{p} f_{i j}-\eta k^{2} G_{\eta} k_{s p} \nabla_{s} f_{i j}\right)-\bar{B}_{i}\left(\frac{1}{2} \nabla_{s}-\eta k^{2} G_{\eta} k_{s p} \nabla_{p}\right) f_{s j}\right] .
$$

Since we neglect effects $\sim \mathrm{O}\left(\lambda_{i} \nabla_{i} \overline{\boldsymbol{u}^{2}}, \lambda^{2} \overline{\boldsymbol{u}^{2}}, \nabla^{2} \overline{\boldsymbol{u}^{2}}\right)$, the term $g_{i j}^{(2)}(\boldsymbol{k}, \omega)$ describes only the contribution to $\gamma$ caused by $\nabla \overline{u^{2}}$, i.e., the effect of inhomogeneity of turbulence, rather than the effect of stratification on $\gamma$. A non-zero contribution to $g_{i j}^{(2)}$ (and to the mean electromotive force) is only from the last two terms in (B3), which are proportional to $f_{s j}$, where $f_{s j} \propto\left(\delta_{s j}-k_{s j}+2 \sigma_{c} k_{s j}\right) \overline{u^{2}}$. Indeed, the first two terms $\propto f_{i j}$ in (B33) (which is a symmetric tensor with respect to the indexes $i$ and $j$ ) cannot contribute to the electromotive force. Here we also took into account that $\int k_{i} \sin \theta d \theta d \varphi=0$. After integration in $\boldsymbol{k}$ and $\omega$ space we arrive at (2.20) for the effective pumping velocity $\gamma$ for $\mathrm{Rm} \ll 1$. For integration over $\omega$, we use the following integrals $\tau_{0} \gg\left(\eta k^{2}\right)^{-1}$ :

$$
\begin{aligned}
& \int_{-\infty}^{\infty} \frac{d \omega}{\left( \pm i \omega+\eta k^{2}\right)\left(\omega^{2}+\tau_{0}^{-2}\right)}=\frac{\pi \tau_{0}}{\tau_{0}^{-1}+\eta k^{2}} \approx \frac{\pi \tau_{0}}{\eta k^{2}}, \\
& \int_{-\infty}^{\infty} \frac{d \omega}{\left(i \omega+\eta k^{2}\right)\left(-i \omega+\eta k^{2}\right)\left(\omega^{2}+\tau_{0}^{-2}\right)}=\frac{\pi \tau_{0}}{\eta k^{2}\left(\tau_{0}^{-1}+\eta k^{2}\right)} \approx \frac{\pi \tau_{0}}{\eta^{2} k^{4}} .
\end{aligned}
$$

Applying the turbulence model of Amir et al. (2017) for arbitrary stratification (see 
also Elperin et al. 1995),

$$
\overline{u_{i}(\boldsymbol{k}) u_{j}(-\boldsymbol{k})}=\frac{\overline{\boldsymbol{u}^{2}} E(k)}{8 \pi\left(k^{2}+\lambda^{2}\right)}\left[\delta_{i j}-\frac{k_{i} k_{j}}{k^{2}}+\frac{i}{k^{2}}\left(\lambda_{i} k_{j}-\lambda_{j} k_{i}\right)+\frac{\lambda^{2}}{k^{2}}\left(\delta_{i j}-\frac{\lambda_{i} \lambda_{j}}{\lambda^{2}}\right)\right],
$$

we show that there are no contributions of $\boldsymbol{\lambda}$ to the effective pumping velocity $\boldsymbol{\gamma}$ of the large-scale magnetic field-even for strong stratifications. In particular, we substitute (B 6) into (B 2), taking into account that $\int k_{p} \sin \theta d \theta d \varphi=0$. Note also that a crosseffect of stratification and inhomogeneity of turbulence, $\left(\lambda_{i} \nabla_{j}-\lambda_{j} \nabla_{i}\right) \overline{\boldsymbol{u}^{2}}$, in the model of the background turbulence does not contribute to the effective pumping velocity $\gamma$ for the same reasons.

\section{Appendix C. Derivation of (2.21) for $\mathbf{R m} \gg 1$ and (3.11) for $\mathrm{Pe} \gg 1$}

For the derivation of (2.21), we perform the following calculation. We use the identity:

$$
\frac{\partial}{\partial t} \overline{b_{i}\left(\boldsymbol{k}_{1}, t\right) u_{j}\left(\boldsymbol{k}_{2}, t\right)}=\overline{\frac{\partial b_{i}\left(\boldsymbol{k}_{1}, t\right)}{\partial t} u_{j}\left(\boldsymbol{k}_{2}, t\right)}+\overline{b_{i}\left(\boldsymbol{k}_{1}, t\right) \frac{\partial u_{j}\left(\boldsymbol{k}_{2}, t\right)}{\partial t}} .
$$

We rewrite the induction equation (2.5) for magnetic fluctuations in $\boldsymbol{k}$ space:

$$
\frac{\partial b_{i}(\boldsymbol{k})}{\partial t}=i k_{p}\left(\int \bar{B}_{p}(\boldsymbol{Q}) u_{i}(\boldsymbol{k}-\boldsymbol{Q}) d \boldsymbol{Q}-\int \bar{B}_{i}(\boldsymbol{Q}) u_{p}(\boldsymbol{k}-\boldsymbol{Q}) d \boldsymbol{Q}\right)+b_{i}^{(\mathrm{N})}(\boldsymbol{k}),
$$

using (A 4)- Here $b_{i}^{(\mathrm{N})}(\boldsymbol{k})$ includes the nonlinear terms. For brevity of notations we omit below the variable $t$ in the functions $\bar{B}_{i}(\boldsymbol{Q}, t), b_{i}(\boldsymbol{k}, t), b_{i}^{(\mathrm{N})}(\boldsymbol{k}, t)$ and $u_{i}(\boldsymbol{k}, t)$. To derive (2.21), we use (C1) and (C2) which yield

$$
\frac{\partial g_{i j}(\boldsymbol{k}, \boldsymbol{R})}{\partial t}=\tilde{J}_{i j}^{(1)}(\boldsymbol{k}, \boldsymbol{R})-\tilde{J}_{i j}^{(2)}(\boldsymbol{k}, \boldsymbol{R})+g_{i j}^{(\mathrm{N})}(\boldsymbol{k}, \boldsymbol{R})
$$

where

$$
\begin{aligned}
\tilde{J}_{i j}^{(1)}(\boldsymbol{k}, \boldsymbol{R})= & i \int\left(k_{p}+K_{p} / 2\right) \bar{B}_{p}(\boldsymbol{Q}) \overline{u_{i}(\boldsymbol{k}+\boldsymbol{K} / 2-\boldsymbol{Q}) u_{j}(-\boldsymbol{k}+\boldsymbol{K} / 2)} \\
& \times \exp (i \boldsymbol{K} \cdot \boldsymbol{R}) d \boldsymbol{K} d \boldsymbol{Q}, \\
\tilde{J}_{i j}^{(2)}(\boldsymbol{k}, \boldsymbol{R})= & i \int\left(k_{p}+K_{p} / 2\right) \bar{B}_{i}(\boldsymbol{Q}) \overline{u_{p}(\boldsymbol{k}+\boldsymbol{K} / 2-\boldsymbol{Q}) u_{j}(-\boldsymbol{k}+\boldsymbol{K} / 2)} \\
& \times \exp (i \boldsymbol{K} \cdot \boldsymbol{R}) d \boldsymbol{K} d \boldsymbol{Q} .
\end{aligned}
$$

Next, we perform calculations that are similar to (A 10) A 12 and A 14 A 18). In particular, we introduce new variables: $\tilde{\boldsymbol{k}}=\left(\tilde{\boldsymbol{k}}_{1}-\tilde{\boldsymbol{k}}_{2}\right) / 2=\boldsymbol{k}-\boldsymbol{Q} / 2$ and $\tilde{\boldsymbol{K}}=\tilde{\boldsymbol{k}}_{1}+\tilde{\boldsymbol{k}}_{2}=$ $\boldsymbol{K}-\boldsymbol{Q}$, and use the Taylor expansion for $|\boldsymbol{Q}| \ll|\boldsymbol{k}|$ and $|\boldsymbol{K}| \ll|\boldsymbol{k}|$, which yield

$$
\begin{aligned}
& \tilde{J}_{i j}^{(1)}(\boldsymbol{k}, \boldsymbol{R})=\bar{B}_{p}\left(i k_{p} f_{i j}+\frac{1}{2} \nabla_{p} f_{i j}\right)-\frac{1}{2} k_{m} \frac{\partial f_{i j}}{\partial k_{p}} \nabla_{p} \bar{B}_{m}+O\left(\nabla^{2}\right) \\
& \tilde{J}_{i j}^{(2)}(\boldsymbol{k}, \boldsymbol{R})=\bar{B}_{i}\left(i k_{p} f_{p j}+\frac{1}{2} \nabla_{p} f_{p j}\right)+\frac{1}{2}\left(f_{p j}-k_{m} \frac{\partial f_{m j}}{\partial k_{p}}\right)\left(\nabla_{p} \bar{B}_{i}\right)+O\left(\nabla^{2}\right) .
\end{aligned}
$$

Therefore, (C2)-C7) yield (2.21) for $\mathrm{Rm} \gg 1$.

For the derivation of (3.11), we perform the following calculation. We use the identity:

$$
\frac{\partial}{\partial t} \overline{n\left(\boldsymbol{k}_{1}, t\right) u_{j}\left(\boldsymbol{k}_{2}, t\right)}=\overline{\frac{\partial n\left(\boldsymbol{k}_{1}, t\right)}{\partial t} u_{j}\left(\boldsymbol{k}_{2}, t\right)}+\overline{n\left(\boldsymbol{k}_{1}, t\right) \frac{\partial u_{j}\left(\boldsymbol{k}_{2}, t\right)}{\partial t}} .
$$


We rewrite equation (3.3) for fluctuations of the particle number density, in $\boldsymbol{k}$ space:

$$
\frac{\partial n(\boldsymbol{k})}{\partial t}=-i k_{p} \int N(\boldsymbol{Q}) u_{p}(\boldsymbol{k}-\boldsymbol{Q}) d \boldsymbol{Q}+n^{(\mathrm{N})}(\boldsymbol{k}),
$$

using (A 4). Here $n^{(\mathrm{N})}(\boldsymbol{k})$ includes the nonlinear terms. For brevity of notations we omit below the variable $t$ in the functions $N(\boldsymbol{Q}, t), n(\boldsymbol{k}, t), n^{(\mathrm{N})}(\boldsymbol{k}, t)$ and $u_{i}(\boldsymbol{k}, t)$. To derive (3.11), we use (C8) and (C9) which yield

$$
\frac{\partial F_{j}(\boldsymbol{k}, \boldsymbol{R})}{\partial t}=\tilde{J}_{j}^{(3)}(\boldsymbol{k}, \boldsymbol{R})+F_{j}^{(\mathrm{N})}(\boldsymbol{k}, \boldsymbol{R}),
$$

where

$$
\begin{aligned}
\tilde{J}_{j}^{(3)}(\boldsymbol{k}, \boldsymbol{R})= & -i \int\left(\boldsymbol{k}_{p}+K_{p} / 2\right) N(\boldsymbol{Q}) \overline{u_{p}(\boldsymbol{k}+\boldsymbol{K} / 2-\boldsymbol{Q}) u_{j}(-\boldsymbol{k}+\boldsymbol{K} / 2)} \\
& \times \exp (i \boldsymbol{K} \cdot \boldsymbol{R}) d \boldsymbol{K} d \boldsymbol{Q} .
\end{aligned}
$$

We perform calculations that are similar to A 10- A 12 and A 14 - A 18), which yield

$$
\tilde{J}_{j}^{(3)}(\boldsymbol{k}, \boldsymbol{R})=-N\left(i k_{p} f_{p j}+\frac{1}{2} \nabla_{p} f_{p j}\right)-\frac{1}{2}\left(f_{p j}-k_{m} \frac{\partial f_{m j}}{\partial k_{p}}\right) \nabla_{p} N .
$$

Therefore, (C8)-C12) yield (3.11) for $\mathrm{Pe} \gg 1$.

\section{REFERENCES}

Akhiezer, A. I. \& Peletminskit, S. V. 1981 Methods of Statistical Physics. Pergamon.

Amir, G., Bar, N., Eidelman, A., Elperin, T., Kleeorin, N. \& Rogachevskit, I. 2017 Turbulent thermal diffusion in strongly stratified turbulence: theory and experiments. Phys. Rev. Fluids 2 (6), 064605.

Armitage, P. J. 2010 Astrophysics of Planet Formation. Cambridge University Press.

Balachandar, S. \& Eaton, J. K. 2010 Turbulent dispersed multiphase flow. Annu. Rev. Fluid Mech. 42, 111-133.

Batchelor, G. K. 1953 The theory of homogeneous turbulence. Cambridge Univ. Press.

Blackman, E. G. \& Field, G. B. 2003 A new approach to turbulent transport of a mean scalar. Phys. Fluids 15 (11), L73-L76.

Brandenburg, A., Gressel, O., Käpylä, P. J., Kleeorin, N., Mantere, M. J. \& RoGACHEVSKII, I. 2012a New scaling for the alpha effect in slowly rotating turbulence. Astrophys. J. 762 (2), 127.

Brandenburg, A., RÄDler, K.-H. \& Kemel, K. 2012b Mean-field transport in stratified and/or rotating turbulence. Astron. Astroph. 539, A35.

Brandenburg, A., RÄDler, K.-H., Rheinhardt, M. \& Käpylä, P. J. 2008 Magnetic diffusivity tensor and dynamo effects in rotating and shearing turbulence. Astroph. J. 676 (1), 740.

Brandenburg, A. \& Subramanian, K. 2005 Astrophysical magnetic fields and nonlinear dynamo theory. Phys. Rep. 417 (1), 1-209.

Buchholz, J., Eidelman, A., Elperin, T., Grünefeld, G., Kleeorin, N., Krein, A. \& Rogachevski, I. 2004 Experimental study of turbulent thermal diffusion in oscillating grids turbulence. Experim. Fluids 36 (6), 879-887.

Caporaloni, M., Tampieri, F., Trombetti, F. \& Vittori, O. 1975 Transfer of particles in nonisotropic air turbulence. J. Atmosph. Sci. 32 (3), 565-568.

Chandrasekhar, S. 1943 Stochastic problems in physics and astronomy. Rev. Modern Phys. $15(1), 1$.

Chassaing, P., Antonia, R. A., Anselmet, F., Joly, L. \& Sarkar, S. 2013 Variable density fluid turbulence. Springer Science \& Business Media, v. 69.

Crowe, C. T., Schwarzkopf, J. D., Sommerfeld, M. \& Tsuji, Y. 2011 Multiphase flows with droplets and particles. CRC press. 
Csanady, G. T. 1980 Turbulent Diffusion in the Environment. Reidel, Dordrecht.

Eidelman, A., Elperin, T., Kleeorin, N., Melnik, B. \& Rogachevskit, I. 2010 Tangling clustering of inertial particles in stably stratified turbulence. Phys. Rev. E 81 (5), 056313.

Eidelman, A., Elperin, T., Kleeorin, N., Rogachevskit, I. \& Sapir-Katiraie, I. 2006 Turbulent thermal diffusion in a multi-fan turbulence generator with imposed mean temperature gradient. Experim. Fluids 40 (5), 744.

Elperin, T., Kleeorin, N., Liberman, M. \& Rogachevskit, I. 2013 Tangling clustering instability for small particles in temperature stratified turbulence. Phys. Fluids 25 (8), 085104 .

Elperin, T., Kleeorin, N. \& Rogachevskit, I. 1995 Dynamics of the passive scalar in compressible turbulent flow: Large-scale patterns and small-scale fluctuations. Phys. Rev. E $52(3), 2617$.

Elperin, T., Kleeorin, N. \& Rogachevski, I. 1996 Turbulent thermal diffusion of small inertial particles. Phys. Rev. Lett. 76 (2), 224.

Elperin, T., KleEorin, N. \& Rogachevskit, I. 1997 Turbulent barodiffusion, turbulent thermal diffusion, and large-scale instability in gases. Phys. Rev. E 55 (3), 2713.

Elperin, T., Kleeorin, N. \& Rogachevskit, I. 1998a Formation of inhomogeneities in twophase low-mach-number compressible turbulent fluid flows. Intern. J. Multiphase Flow 24 (7), 1163-1182.

Elperin, T., Kleeorin, N. \& Rogachevskit, I. 1998b Dynamics of particles advected by fast rotating turbulent fluid flow: fluctuations and large-scale structures. Phys. Rev. Lett. 81 (14), 2898-2901.

Elperin, T., Kleeorin, N., Rogachevskit, I. \& Sokoloff, D. 2000 Passive scalar transport in a random flow with a finite renewal time: mean-field equations. Phys. Rev. E 61 (3), 2617.

Elperin, T., Kleeorin, N., Rogachevskit, I. \& Sokoloff, D. 2001 Mean-field theory for a passive scalar advected by a turbulent velocity field with a random renewal time. Phys. Rev. E 64 (2), 026304.

Elperin, T., Kleeorin, N., Rogachevskit, I. \& Zilitinkevich, S. 2002 Formation of largescale semiorganized structures in turbulent convection. Phys. Rev. E 66 (6), 066305.

Elperin, T., Kleeorin, N., Rogachevskit, I. \& Zilitinkevich, S. S. 2006 Tangling turbulence and semi-organized structures in convective boundary layers. Boundary-layer Meteorol. 119 (3), 449-472.

Frisch, U. 1995 Turbulence: the Legacy of A. N. Kolmogorov. Cambridge university press.

Haugen, N. E. L., Kleeorin, N., Rogachevskit, I. \& Brandenburg, A. 2012 Detection of turbulent thermal diffusion of particles in numerical simulations. Phys. Fluids 24 (7), 075106.

Hodgson, L. S. \& Brandenburg, A. 1998 Turbulence effects in planetesimal formation. Astron. Astrophys. 330, 1169-1174.

Hubbard, A. 2015 Turbulent thermal diffusion: a way to concentrate dust in protoplanetary discs. Mon. Not. Roy. Astron. Soc. 456 (3), 3079-3089.

Käpylä, P.J., Brandenburg, A., Kleeorin, N., Mantere, M.J. \& Rogachevskit, I. 2012 Negative effective magnetic pressure in turbulent convection. Mon. Not. Roy. Astron. Soc. 422 (3), 2465-2473.

Kleeorin, N. \& Rogachevskit, I. 2003 Effect of rotation on a developed turbulent stratified convection: The hydrodynamic helicity, the $\alpha$ effect, and the effective drift velocity. Phys. Rev. E 67 (2), 026321.

Kleeorin, N. \& Rogachevskit, I. 2018 Generation of large-scale vorticity in rotating stratified turbulence with inhomogeneous helicity: mean-field theory. J. Plasma Phys. 84 (3), 735840303.

Klemorin, N., Rogachevskit, I. \& Ruzmaikin, A. 1990 Magnetic force reversal and instability in a plasma with advanced magnetohydrodynamic turbulence. Sov. Phys. JETP 70, 878883.

Krause, F. \& RÄDler, K.-H. 1980 Mean-Field Magnetohydrodynamics and Dynamo Theory. Pergamon.

MAXEY, M. R. 1987 The gravitational settling of aerosol particles in homogeneous turbulence and random flow fields. J. Fluid Mech. 174, 441-465. 
McComb, W. D. 1990 The Physics of Fluid Turbulence. Clarendon.

Mitra, D., Haugen, N. E. L. \& Rogachevskit, I. 2018 Turbophoresis in forced inhomogeneous turbulence. Eur. Phys. J. Plus 133 (6), 35.

Moffatt, H. K. 1978 Field Generation in Electrically Conducting Fluids. Cambridge University Press.

Monin, A. S. \& Yaglom, A. M. 2013 Statistical Fluid Mechanics. Courier Corporation.

Orszag, S. A. 1970 Analytical theories of turbulence. J. Fluid Mech. 41 (2), 363-386.

Pandya, R. V. R. \& Mashayek, F. 2002 Turbulent thermal diffusion and barodiffusion of passive scalar and dispersed phase of particles in turbulent flows. Phys. Rev. Lett. 88 (4), 044501.

PARker, E. N. 1979 Cosmical Magnetic Fields: Their Origin and Their Activity. Oxford University Press.

Piterbarg, L. \& Ostrovskit, A. 2013 Advection and Diffusion in Random Media. Springer Science \& Business Media.

Pouquet, A., Frisch, U. \& LÉOrat, J. 1976 Strong mhd helical turbulence and the nonlinear dynamo effect. J. Fluid Mech. 77 (2), 321-354.

PRIEST, E. R. 1982 Solar Magnetohydrodynamics. Reidel Publ., Dordrecht.

Rädler, K.-H., Brandenburg, A., Del Sordo, F. \& Rheinhardt, M. 2011 Mean-field diffusivities in passive scalar and magnetic transport in irrotational flows. Phys. Rev. E 84 (4), 046321.

RÄDler, K.-H., KleEorin, N. \& Rogachevskit, I. 2003 The mean electromotive force for mhd turbulence: the case of a weak mean magnetic field and slow rotation. Geophys. Astroph. Fluid Dyn. 97 (3), 249-274.

ReEks, M. W. 1983 The transport of discrete particles in inhomogeneous turbulence. J. Aerosol Sci. 14 (6), 729-739.

REeKs, M. W. 1992 On the continuum equations for dispersed particles in nonuniform flows. Phys. Fluids 4 (6), 1290-1303.

REEKs, M. W. 2005 On model equations for particle dispersion in inhomogeneous turbulence. Intern. J. Multiph. Flow 31 (1), 93-114.

Roberts, P. H. \& SowArd, A. M. 1975 A unified approach to mean field electrodynamics. Astron. Nachr. 296 (2), 49-64.

Rogachevskit, I. \& Kleeorin, N. 2000 Electromotive force for an anisotropic turbulence: Intermediate nonlinearity. Phys. Rev. E 61 (5), 5202.

Rogachevskit, I. \& Kleeorin, N. 2001 Nonlinear turbulent magnetic diffusion and mean-field dynamo. Phys. Rev. E 64 (5), 056307.

Rogachevskit, I. \& Kleeorin, N. 2003 Electromotive force and large-scale magnetic dynamo in a turbulent flow with a mean shear. Phys. Rev. E 68 (3), 036301.

Rogachevskit, I. \& Kleeorin, N. 2004 Nonlinear theory of a "shear-current" effect and mean-field magnetic dynamos. Phys. Rev. E 70 (4), 046310.

Rogachevskit, I. \& Kleeorin, N. 2006 Small-scale magnetic buoyancy and magnetic pumping effects in a turbulent convection. Geophys. Astrophys. Fluid Dyn. 100 (3), 243-263.

Rogachevskit, I. \& Kleeorin, N. 2007 Magnetic fluctuations and formation of large-scale inhomogeneous magnetic structures in a turbulent convection. Phys. Rev. E 76 (5), 056307.

Rogachevskit, I. \& Kleeorin, N. 2018 Mean-field theory of differential rotation in density stratified turbulent convection. J. Plasma Phys. 84 (2), 735840201.

Rogachevskit, I., Kleeorin, N., Brandenburg, A. \& Eichler, D. 2012 Cosmic-ray currentdriven turbulence and mean-field dynamo effect. Astrophys. J. 753 (1), 6.

Rogachevskit, I., Kleeorin, N., Käpylä, P. J. \& Brandenburg, A. 2011 Pumping velocity in homogeneous helical turbulence with shear. Phys. Rev. E 84 (5), 056314.

Rogachevskit, I., Ruchayskiy, O., Boyarsky, A., Fröhlich, J., Kleeorin, N., Brandenburg, A. \& Schober, J. 2017 Laminar and turbulent dynamos in chiral magnetohydrodynamics-i: Theory. Astroph. J. 846 (2), 153.

Rüdiger, G., Kitchatinov, L. L. \& Hollerbach, R. 2013 Magnetic Processes in Astrophysics: theory,simulations, experiments. Wiley-VCH, Weinheim.

Ruzmaikin, A., Shukurov, A. \& Sokoloff, D. 1988 Magnetic Fields of Galaxies. Kluver Acad. Publ.

Schrinner, M., Rädler, K.-H., Schmitt, D., Rheinhardt, M. \& Christensen, U. R. 2005 
Mean-field view on rotating magnetoconvection and a geodynamo model. Astron. Nachr. 326 (3-4), 245-249.

Schrinner, M., RÄdler, K.-H., Schmitt, D., Rheinhardt, M. \& Christensen, U. R. 2007 Mean-field concept and direct numerical simulations of rotating magnetoconvection and the geodynamo. Geophys. Astroph. Fluid Dyn. 101 (2), 81-116.

Sofiev, M., Sofieva, V., Elperin, T., Kleeorin, N., Rogachevskis, I. \& Zilitinkevich, S. S. 2009 Turbulent diffusion and turbulent thermal diffusion of aerosols in stratified atmospheric flows. J. Geophys. Res. 114 (D18).

van Aartrijk, M. \& Clercx, H. J. H. 2008 Preferential concentration of heavy particles in stably stratified turbulence. Phys. Rev. Lett. 100 (25), 254501.

Yokoi, N. \& BRAndenBurG, A. 2016 Large-scale flow generation by inhomogeneous helicity. Phys. Rev. E 93 (3), 033125.

Yokoi, N. \& Yoshizawa, A. 1993 Statistical analysis of the effects of helicity in inhomogeneous turbulence. Phys. Fluids 5 (2), 464-477.

Zaichik, L. I., Alipchenkov, V. M. \& Sinaiski, E. G. 2008 Particles in Turbulent Flows. John Wiley \& Sons.

Zeldovich, Ya. B., Ruzmaikin, A. A. \& Sokoloff, D. D. 1990 The Almighty Chance. World Scientific.

Zeldovich, Ya. B., Ruzmaikin, A. A. \& Sokolov, D. D. 1983 Magnetic Fields in Astrophysics. Gordon and Breach Science Publishers.

Zilitinkevich, S. S., Elperin, T., Kleeorin, N., Lvov, V. \& Rogachevskit, I. 2009 Energyand flux-budget turbulence closure model for stably stratified flows. part ii: the role of internal gravity waves. Boundary-layer Meteorology 133 (2), 139-164.

Zilitinkevich, S. S., Elperin, T., Kleeorin, N., Rogachevskit, I. \& Esau, I. 2013 A hierarchy of energy-and flux-budget (efb) turbulence closure models for stably-stratified geophysical flows. Boundary-layer Meteorol. 146 (3), 341-373. 